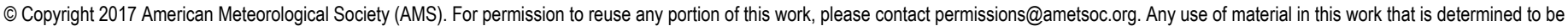

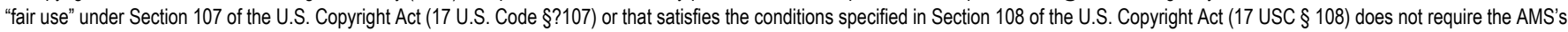

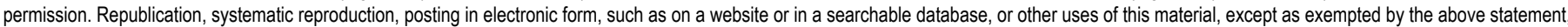

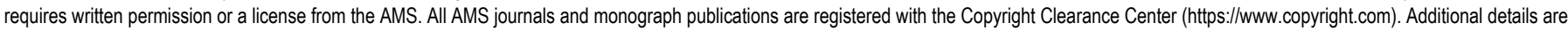
provided in the AMS Copyright Policy statement, available on the AMS website (https://www.ametsoc.org/PUBSCopyrightPolicy).

\title{
REVIEW
}

\section{The Extratropical Transition of Tropical Cyclones. Part I: Cyclone Evolution and Direct Impacts}

\author{
Clark Evans, ${ }^{\mathrm{a}}$ Kimberly M. WoOd, ${ }^{\mathrm{b}}$ Sim D. Aberson, ${ }^{\mathrm{c}}$ Heather M. ArChambault, ${ }^{\mathrm{d}}$

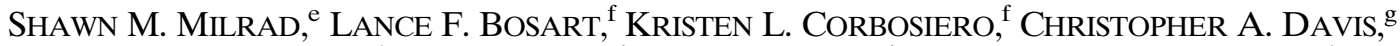

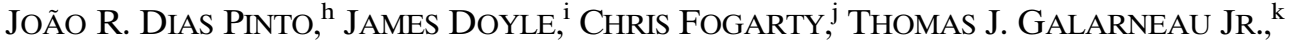

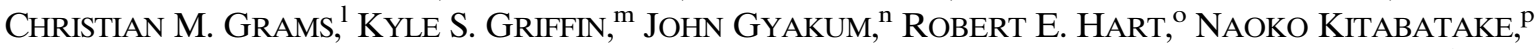 \\ Hilke S. Lentink ${ }^{\mathrm{q}}{ }^{\mathrm{R}}$ Ron MCTAGgart-COWAN,${ }^{\mathrm{r}}$ WILliam PERRIE, ${ }^{\mathrm{s}}$ Julian F. D. Quinting, ${ }^{\mathrm{t}}$ \\ Carolyn A. Reynolds, ${ }^{\mathrm{i}}$ Michael Riemer, ${ }^{\mathrm{u}}$ Elizabeth A. Ritchie, ${ }^{\mathrm{v}}$ YuJuAn Sun, ${ }^{\mathrm{s}}$ ANd FuQing Zhang ${ }^{\mathrm{w}}$ \\ ${ }^{\text {a }}$ University of Wisconsin-Milwaukee, Milwaukee, Wisconsin \\ ${ }^{\mathrm{b}}$ Mississippi State University, Mississippi State, Mississippi \\ ${ }^{\mathrm{c}}$ NOAA/Atlantic Oceanographic and Meteorological Laboratory/Hurricane Research Division, Miami, Florida \\ ${ }^{\mathrm{d}}$ NOAA/Climate Program Office, Silver Spring, Maryland \\ ${ }^{\mathrm{e}}$ Embry-Riddle Aeronautical University, Daytona Beach, Florida \\ ${ }^{\mathrm{f}}$ University at Albany, State University of New York, Albany, New York \\ ${ }^{\mathrm{g}}$ National Center for Atmospheric Research, Boulder, Colorado \\ ${ }^{\mathrm{h}}$ University of São Paulo, São Paulo, Brazil \\ ${ }^{\mathrm{i}}$ Naval Research Laboratory, Monterey, California \\ ${ }^{\mathrm{j}}$ Canadian Hurricane Center, Dartmouth, Nova Scotia, Canada \\ ${ }^{\mathrm{k}}$ The University of Arizona, Tucson, Arizona \\ ${ }^{1}$ Institute for Atmospheric and Climate Science, ETH Zurich, Zurich, Switzerland \\ ${ }^{\mathrm{m}}$ RiskPulse, Madison, Wisconsin \\ ${ }^{\mathrm{n}}$ Mc Gill University, Montreal, Quebec, Canada \\ ${ }^{\circ}$ Florida State University, Tallahassee, Florida \\ ${ }^{\mathrm{p}}$ Meteorological College, Kashiwa, Chiba, Japan \\ ${ }^{\mathrm{q}}$ Karlsruhe Institute of Technology, Karlsruhe, Germany \\ ${ }^{\mathrm{r}}$ Environment and Climate Change Canada, Dorval, Quebec, Canada \\ ${ }^{\mathrm{s}}$ Bedford Institute of Oceanography, Dartmouth, Nova Scotia, Canada \\ ${ }^{\mathrm{t}}$ School of Earth, Atmosphere and Environment, and ARC Centre of Excellence for \\ Climate System Science, Monash University, Clayton, Victoria, Australia \\ u Johannes Gutenberg-Universität Mainz, Mainz, Germany \\ ${ }^{\mathrm{v}}$ University of New South Wales, Canberra, Australia \\ ${ }^{\mathrm{w}}$ The Pennsylvania State University, University Park, Pennsylvania
}

(Manuscript received 7 February 2017, in final form 8 August 2017)

ABSTRACT

Extratropical transition (ET) is the process by which a tropical cyclone, upon encountering a baroclinic environment and reduced sea surface temperature at higher latitudes, transforms into an extratropical cyclone. This process is influenced by, and influences, phenomena from the tropics to the midlatitudes and from the meso- to the planetary scales to extents that vary between individual events. Motivated in part by recent high-impact and/or extensively observed events such as North Atlantic Hurricane Sandy in 2012 and western North Pacific Typhoon Sinlaku in 2008, this review details advances in understanding and predicting ET since the publication of an earlier review in 2003. Methods for diagnosing ET in reanalysis, observational, and model-forecast datasets are discussed. New climatologies for the eastern North Pacific and southwest Indian Oceans are presented alongside updates to western North Pacific and North Atlantic Ocean climatologies. Advances in understanding and, in some cases, modeling the direct impacts of ET-related wind, waves, and precipitation are noted. Improved understanding of structural evolution throughout the transformation stage of ET fostered in large part by novel aircraft observations collected in several recent ET events is highlighted. Predictive skill for operational and numerical model ET-related forecasts is discussed along with environmental factors influencing posttransition cyclone structure and evolution. Operational ET forecast and analysis practices and challenges are detailed. In particular, some 
challenges of effective hazard communication for the evolving threats posed by a tropical cyclone during and after transition are introduced. This review concludes with recommendations for future work to further improve understanding, forecasts, and hazard communication.

\section{Introduction}

In October 2012, Hurricane Sandy drove a devastating storm surge in excess of $2 \mathrm{~m}$ into the northeastern U.S. coastline, tore down trees and power lines that left millions without electricity, and dumped over $900 \mathrm{~mm}$ of snow (Blake et al. 2013). As Sandy approached the coast, it acquired structural characteristics consistent with both tropical and extratropical cyclones, with an intact inner-tropical cyclone (TC) warm core embedded within an expansive outer-core wind field (Blake et al. 2013). Contributions from both tropical and baroclinic energy sources caused Sandy to reintensify as it approached the coastline (Galarneau et al. 2013; Shin and Zhang 2017). The TC followed an atypical track northwestward toward the Northeast United States, rather than out to sea, fostered by interaction with an upstream trough (Barnes et al. 2013; Qian et al. 2016) of the type identified by Fujiwhara (1931), the practical predictability of which depended on the modeling system (Bassill 2014; Magnusson et al. 2014; Torn et al. 2015). Sandy tested existing infrastructure for hazard communication (NOAA 2013; Blake et al. 2013) and posed challenges related to risk perception (Meyer et al.2014) due to its atypical track and forecast structure (Munsell and Zhang 2014) near landfall. Few TCs produce such a broad range of impacts, but Sandy was not ordinary. Rather, Sandy is a dramatic example of the direct impacts, structural evolution, and forecast challenges associated with TCs that become extratropical cyclones, a process known as extratropical transition (ET; Jones et al. 2003).

Tropical cyclones gain energy from warm ocean waters through evaporation and subsequent latent heat release by deep, moist convection. The storm develops a warm core as a result, with the strongest winds near the surface that decrease in strength with height. The wind, precipitation, and temperature fields become more axisymmetric as the TC matures. Conversely, extratropical cyclones are driven by comparatively large temperature and moisture gradients. Within these baroclinic environments, frontal boundaries separate warm, moist air from cool, dry air, resulting in highly asymmetric energy distributions to drive wind and rainfall. In addition, wind speed increases with height due to the cold-core structure of these systems. During ET, the deep warm core associated with the TC becomes shallow and is often replaced by a cold-core, asymmetric structure (e.g., Evans and Hart 2003; Hart et al. 2006), including the development of surface fronts (Klein et al. 2000). This evolution occurs as the TC moves poleward into a baroclinic environment characterized by the aforementioned temperature and moisture gradients as well as increased vertical wind shear, reduced sea surface temperature (SST), and an increasing Coriolis parameter (Fig. 1). Only a subset of TCs complete ET and become fully extratropical, yet even a cyclone that only begins ET can directly produce hazards (such as Hurricane Sandy) and/or generate hazards downstream [e.g., Hurricane Katia in 2011 as described by Grams and Blumer (2015); Typhoon Nabi in 2005 as described by Harr et al. (2008)].

An earlier review (Jones et al. 2003) provided a thencurrent synthesis of the fundamental understanding of ET and its direct impacts. The paper also outlined significant ET-related forecast challenges and research needs that had yet to be addressed. Focusing on ET itself and the direct impacts of transitioning cyclones, the present review documents the extensive research that addresses how those needs have been met in the most recent decade and a half. This review also identifies questions that remain unanswered as well as potential avenues for future research that have been motivated by recent investigations. Section 2 discusses efforts toward a universal definition of, and classifiers for, ET. Section 3 documents the development of, and additions to, new and existing ET climatologies and looks to how ET climatology may change in the future. Section 4 describes the updated understanding of direct impacts associated with wind, waves, and precipitation. Jones et al. (2003) stressed the necessity of improving use of existing observations and exploiting new capabilities for understanding ET itself, as well as forecasting the phenomenon, and section 5 summarizes progress in and ongoing needs for both. Section 6 documents advances in the forecasting and analysis of ET. Finally, this review concludes with recommendations for future research. As noted above, this review focuses on ET and its direct impacts. A companion article (Keller et al. 2018, manuscript submitted to Mon. Wea. Rev., hereafter Part II)

¿ Denotes content that is immediately available upon publication as open access.

Corresponding author: Dr. Clark Evans, evans36@uwm.edu 


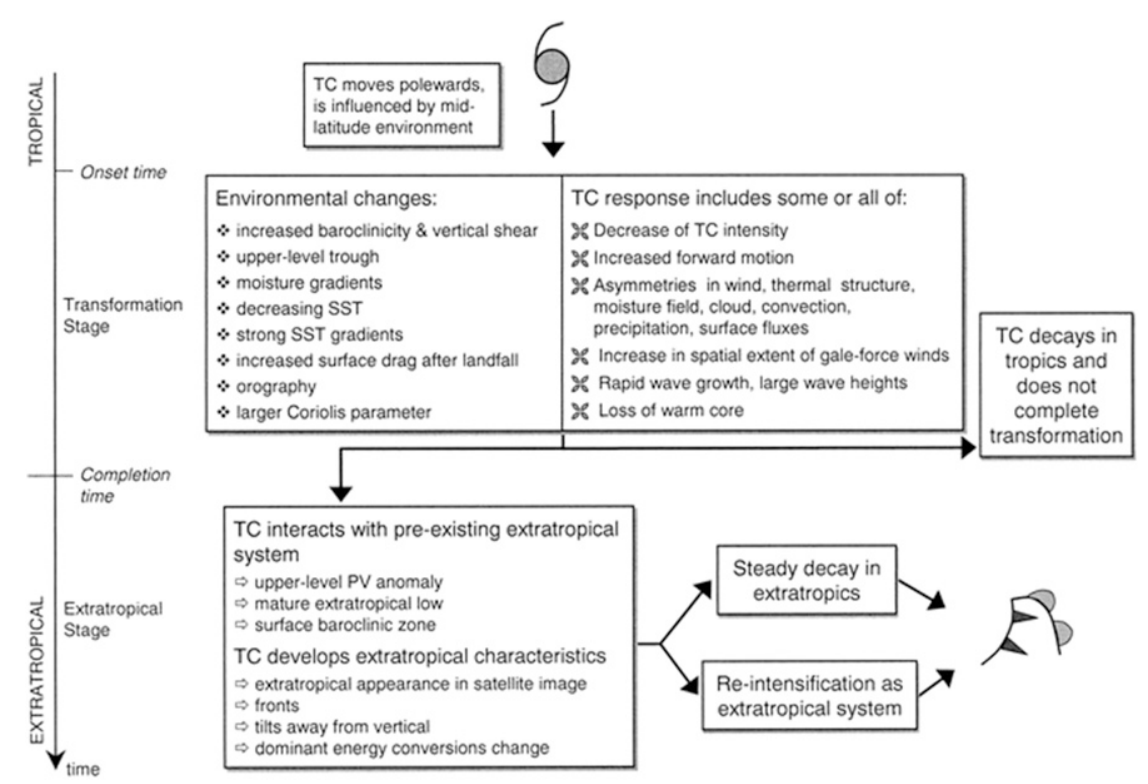

FIG. 1. A two-stage ET classification based on Klein et al. (2000). The onset and completion times correspond to the definitions of Evans and Hart (2003). The "tropical" and "extratropical" labels indicate approximately how the system would be regarded by an operational forecast center. Figure reproduced from Jones et al. (2003, their Fig. 11).

covers research progress related to ET's downstream impacts, including downstream cyclogenesis, cyclone evolution after becoming extratropical, effects on the midlatitude flow and predictability, and phenomena such as predecessor rain events.

\section{Classifiers}

Jones et al. (2003) discussed the need to develop improved conceptual models of, and diagnostic tools for, ET, with a particular focus toward the development of a universal ET definition that can be applied broadly (including operationally). Since their review, the cyclone phase space (CPS; Hart 2003) has become widely accepted in the research and operational communities as a means of diagnosing ET in reanalysis, observational, and model-forecast datasets (e.g., Evans and Hart 2003). The first CPS parameter assesses the thermal symmetry of the TC. Symmetry is defined as the difference in 900 600-hPa geopotential thickness between the right and left sides of the TC relative to its direction of motion, a value known as $B$. A mature, axisymmetric TC exhibits $B$ values of less than $10 \mathrm{~m}$, whereas a cyclone with mature or developing frontal structures is characterized by $B$ values exceeding $10 \mathrm{~m}$. The $10-\mathrm{m}$ threshold approximately corresponds to an across-cyclone layer-mean temperature gradient of $0.85^{\circ} \mathrm{C}$. The second CPS parameter estimates the thermal wind between 900 and $600 \mathrm{hPa}$ $\left(-V_{T}^{L}\right)$, computed from the change in the horizontal geopotential height gradient with height. Tropical cyclones are warm-core systems characterized by a positive geopotential thickness anomaly near the center, resulting in a positive value of $-V_{T}^{L}$. Extratropical cyclones are generally cold-core systems characterized by a negative geopotential thickness anomaly near the center, producing a negative value of $-V_{T}^{L}$. A third CPS parameter $\left(-V_{T}^{U}\right)$ estimates thermal wind in the same manner but between 600 and $300 \mathrm{hPa}$. Similar metrics for determining ET were obtained by Satake et al. (2013) in the context of a broader TC tracking algorithm.

Within the CPS framework, ET begins when $B$ exceeds the empirically derived value of $10 \mathrm{~m}$ and ends when $-V_{T}^{L}$ becomes negative (indicating a cold-core thermal structure; Evans and Hart 2003). Both $B$ and $-V_{T}^{L}$ are computed from averages taken within $500 \mathrm{~km}$ of the cyclone's center. The CPS parameters are often derived from numerical weather prediction (NWP) model analyses and forecasts, but CPS applications have been expanded to reanalysis datasets (e.g., Wood and Ritchie 2014a; Hodges et al. 2017; Zarzycki et al. 2017) and, in testing, polar-orbiting satellite retrievals as well. Despite its widespread use, the CPS is not without its limitations: it does not resolve a cyclone's inner-core structure, and it relies on either NWP products, reanalysis datasets, or satellite retrievals.

Because of these CPS limitations, several studies have evaluated other metrics for ET diagnosis, primarily in retrospective analyses, but with an eye toward potential 
forecast applications. For example, the temperature contrast between the cyclone's inner core and external environment was used by Garde et al. (2010) to classify both tropical transition and extratropical transition events. A more focused evaluation was conducted by Kofron et al. (2010a), who examined 82 recurving TCs in the North Atlantic (NATL) and western North Pacific (WNP) from 2003 to 2006. Tropical cyclones were classified based upon whether they reintensified (e.g., decreased minimum sea level pressure) after, dissipated (e.g., increased minimum sea level pressure) after, or recurved without becoming extratropical. Tropical cyclones that reintensified were further distinguished based upon their post-ET thermal structure (cold core vs warm seclusion) and their translation into the "northwest" or "northeast" synoptic-scale midlatitude flow regimes of Harr and Elsberry (2000). Metrics such as scalar frontogenesis (Harr and Elsberry 2000), the CPS (Hart 2003), and an open wave at $500 \mathrm{hPa}$ (Demirci et al. 2007) were unable to reliably distinguish between recurving TCs that did and did not become extratropical, independent of their posttransition evolution. Similar findings for the scalar frontogenesis and open wave metrics were obtained by Wang et al. (2012).

Jones et al. (2003) advocated for an evaluation of the utility of potential vorticity (PV) toward diagnosing ET in both numerical models and observations. In conducting such an evaluation, Kofron et al. (2010b) demonstrated that TCs that begin the transformation stage of ET experience a decrease in midtropospheric PV as the cyclone weakens, whereas the subset of TCs that complete ET experience a subsequent rapid increase in midtropospheric PV as the TC interacts with a midlatitude trough. In their study, PV on the 330-K isentropic surface, which is typically located in the midtroposphere prior to ET and in the upper troposphere after, was a good discriminator of ET versus recurvature without ET and of intensity change after a cyclone becomes extratropical (Kofron et al. 2010b). All 82 TCs in the Kofron et al. (2010b) dataset exhibited a minimum value of $330-\mathrm{K}$ isentropic PV averaged within $500 \mathrm{~km}$ of the center as they moved into the midlatitudes, and the time at which this value became less than an empirical threshold value was tested as a metric for ET onset. For a threshold value of 1.6 PVU (1 PVU $=10^{-6} \mathrm{~K} \mathrm{~kg}^{-1} \mathrm{~m}^{2} \mathrm{~s}^{-1}$ ), the ET completion forecast success rate for reintensifying cases was $94.3 \%$ with a $27.6 \%$ false alarm rate. The 1.6-PVU metric also reliably determined ET onset for two WNP cases studied by Wang et al. (2012); however, substantial timing variability existed between the four datasets considered, and Li and Wang (2013) found this metric was unable to reliably determine ET completion and extratropical phase intensity change in higher-resolution datasets. Thus, more work is necessary to assess the reliability of the 1.6-PVU threshold using datasets of varying resolution and quality.

\section{Climatologies}

An important application of ET classifiers is for the development of new and/or extension of existing ET climatologies. At the time of Jones et al.'s (2003) publication, at least partial ET climatologies existed for the NATL (Hart and Evans 2001), WNP (Klein et al. 2000), western South Pacific (Sinclair 2002), and west coast of Australia (Foley and Hanstrum 1994). In general, these reflect basins where strong winds and heavy rains associated with ET events preferentially impact some of the most developed nations in the world and/or where ET is a climatologically frequent occurrence. The advent of new ET classifiers, particularly the CPS, has, in part, led to the development of new ET climatologies for the eastern North Pacific (ENP) and southwest Indian Ocean (SWIO) basins. It has also led to the development of updated and/or expanded ET climatologies for the NATL and WNP. A synthesis of these climatologies is provided in Fig. 2. Sections 3a-e discuss the results of these studies in greater depth, concluding with a discussion of projected future changes in the global ET climatology.

\section{a. Western North Pacific}

Klein et al. (2000) examined 30 ET cases over a 4-yr period, a relatively brief span of time, to develop a three-dimensional conceptual model of the transformation stage (Fig. 3). Since then, several studies have assessed WNP ET cases over multiple decades. Approximately $40 \%$ (for the period 1979-2004; Kitabatake 2011) of WNP TCs within the Japan Meteorological Agency best track database became extratropical prior to dissipation (Fig. 4); a slightly smaller percentage (36.5\%, for the period 1979-2009) recurved while becoming extratropical (Archambault et al. 2013). Of the subset of recurving WNP TCs that underwent ET, $42.5 \%$ reintensified after completing ET (Archambault et al. 2013, their Fig. 7). Most (91\%) WNP TCs become extratropical with a minimum central sea level pressure greater than $970 \mathrm{hPa}$ (Kitabatake 2011); however, a small percentage $(9 \%)$ of TCs become extratropical with central pressures less than $970 \mathrm{hPa}$, particularly from September to November. WNP TCs that become extratropical tend to be stronger and larger at recurvature from September to November than at other times of the year (Archambault et al. 2013). Further study is necessary, however, to understand the causes of 

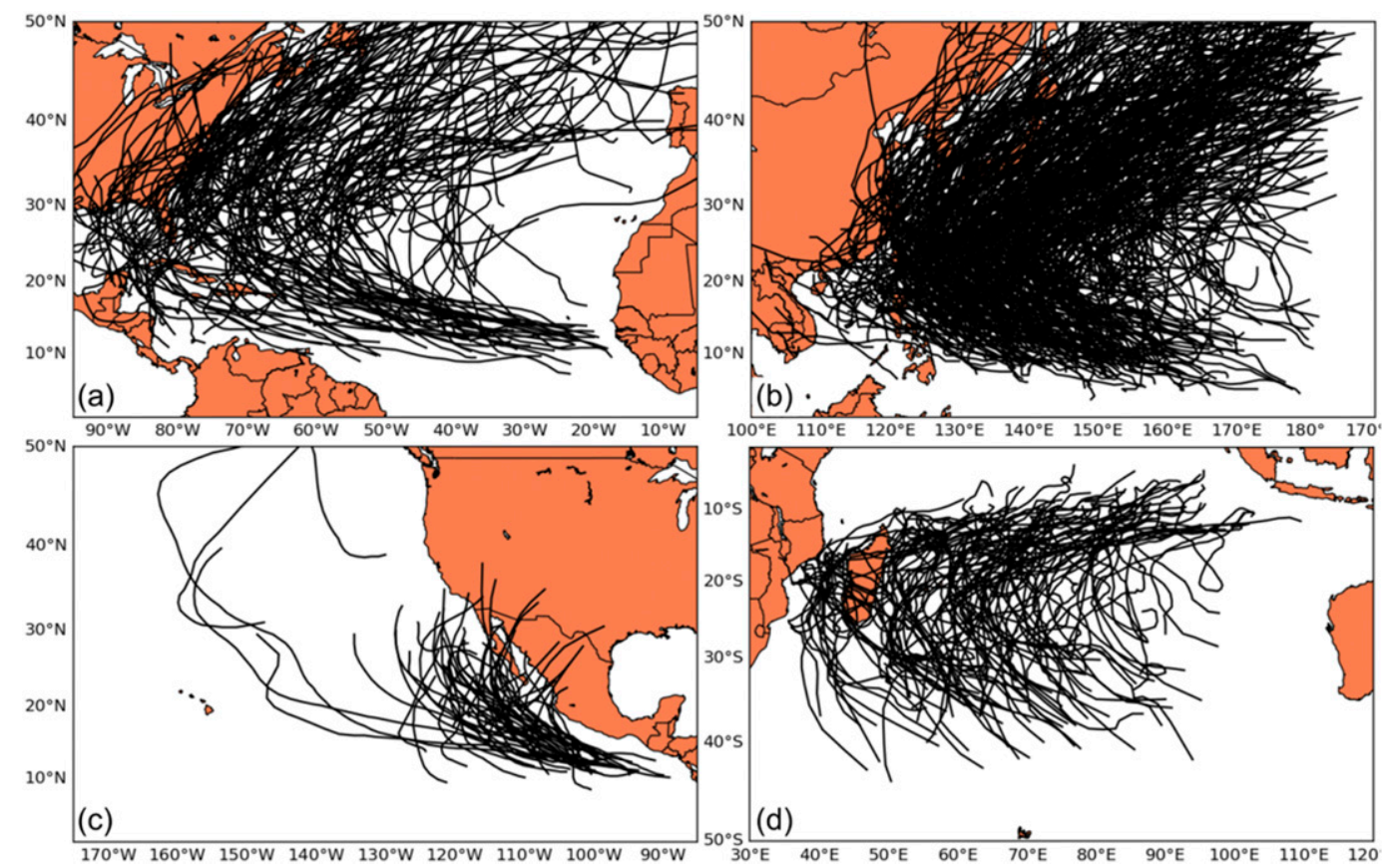

FIG. 2. Tracks of TCs that completed the transformation stage of ET for the (a) NATL [1981-2010; ET designations from HURDAT2 best track data, described in Landsea and Franklin (2013)], (b) WNP (1981-2010; ET designations from Japan Meteorological Agency best track data), (c) ENP [1971-2012; reanalysis-derived CPS ET designations by Wood and Ritchie (2014a)], and (d) SWIO [1987-2013; reanalysis-derived ET designations subjectively determined by Griffin and Bosart (2014)]. No attempt is made to account for ET classification practice differences between operational centers or the historical evolution of ET classification practices at these centers.

this seasonality in both size and intensity after becoming extratropical.

Extratropical transition is not evenly distributed throughout the WNP typhoon season, with the largest number of events found in September (Klein et al. 2000; Kitabatake 2011; Archambault et al. 2013; Quinting and Jones 2016). Note that this contrasts with Jones et al. (2003), who identified August as the peak month for WNP ET events based on Joint Typhoon Warning Center best track data. Although TC frequency increases from June to August, the ratio of TCs that complete ET [based on the Evans and Hart (2003) definition described in section 2] to all TCs is limited to 25\%-35\% during these months (e.g., Kitabatake 2011; Archambault et al. 2013), likely due to the lower probability of a TC interacting with the midlatitude flow associated with the climatological poleward retreat of the polar jet in boreal summer. September is also the month in which the greatest percentage of recurving WNP TCs reintensify after becoming extratropical (Klein et al. 2000; Archambault et al. 2013). The ET frequency varies both between and within WNP typhoon seasons: it occurs less frequently when the subtropical high is anomalously strong, such that TCs are preferentially steered toward Asia rather than recurving toward higher latitudes (Song et al. 2013). A seasonal influence on ET track is also observed, wherein TCs undergoing recurvature and ET tend to recurve more sharply and at lower latitudes in May and October-December than from June to September (Archambault et al. 2013).

\section{b. North Atlantic}

Whereas the initial ET climatology for the WNP only examined cases during a 4-yr period, the initial ET climatology for the NATL (Hart and Evans 2001) examined cases during a much longer period (1950-96). Consequently, research has focused upon expanding, rather than revising or updating, the NATL ET climatology. Specifically, the CPS has been applied to quantify time to ET completion [based on the Evans and Hart (2003) definition described in section 2], post-ET thermal structure, and post-ET intensity change for NATL ET events (Hart et al. 2006). Slowtransitioning TCs were more intense and larger as ET began than their fast-transitioning counterparts. Post-ET weakening was approximately twice as likely as post-ET intensification, whereas post-ET cold-core thermal structure was more than twice as likely as post-ET warm-seclusion thermal structure. Post-ET 


\section{Conceptual Model of Transformation Stage of ET in the Western North Pacific}

(a) Infrared Imagery
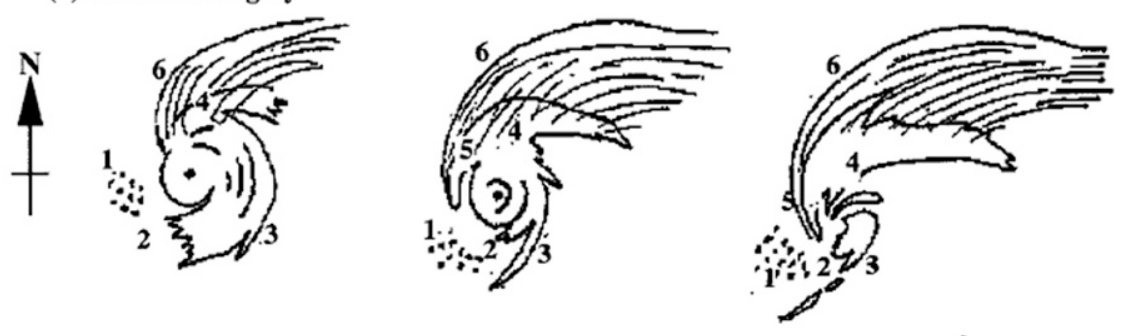

(b) Plan View (Storm Relative Trajectories)
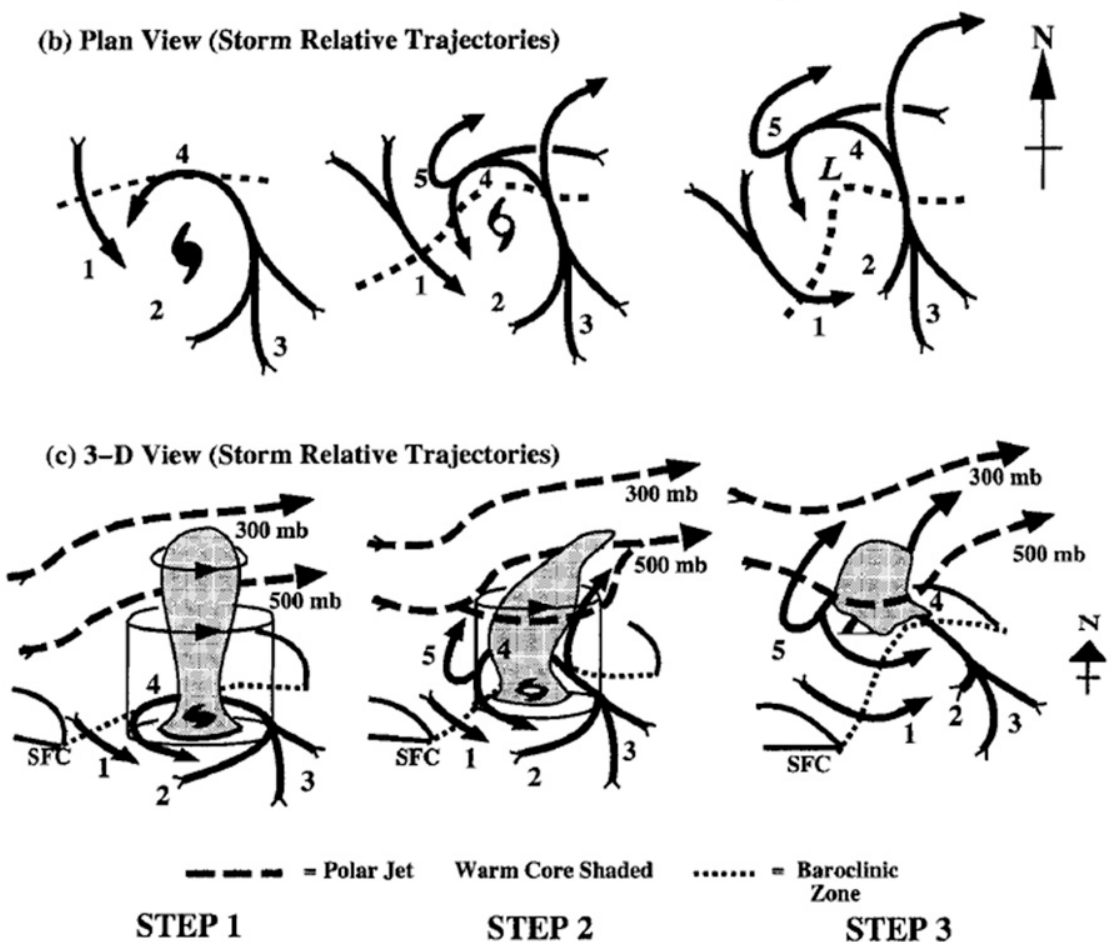

FIG. 3. Conceptual model of the transformation stage of ET in the western North Pacific. Step 1 represents the commencement of the transformation stage, step 2 represents the TC encountering the baroclinic zone, and step 3 represents the TC becoming embedded within the baroclinic zone. Figure reproduced from Klein et al. (2000, their Fig. 5).

warm-seclusion cyclones were larger and were located closer to North America as ET began than their postET cold-core counterparts. However, more research is needed to quantify the effects of TC size and intensity on the outcome of ET as well as what factors govern post-ET intensity change.

\section{c. Eastern North Pacific}

Because of the warm-season climatology of the subtropical ENP, and in contrast to the WNP and NATL, an ENP ET climatology did not exist at the time that Jones et al. (2003) was published. During the North American monsoon (e.g., Adams and Comrie 1997; Vera et al. 2006), the subtropical ridge expands and strengthens north of the ENP main development region. This strong ridge directly reduces the likelihood of midlatitude troughs interacting with ENP TCs, and thus ET likelihood, for most of the hurricane season (e.g., Allard 1984; Corbosiero et al. 2009; Wood and Ritchie 2013, 2014a). Further, ENP TCs rarely survive poleward of $25^{\circ} \mathrm{N}$ due to the cold California Current and climatologically low SST west of Baja California (Corbosiero et al. 2009). As the monsoon wanes late in the season, however, strong midlatitude troughs extend far enough south to potentially interact with TCs and cause them to recurve toward the north and east (Farfàn 2004; Corbosiero et al. 2009; Ritchie et al. 2011; Wood and Ritchie 2013), bringing heavy precipitation to the southwestern United 


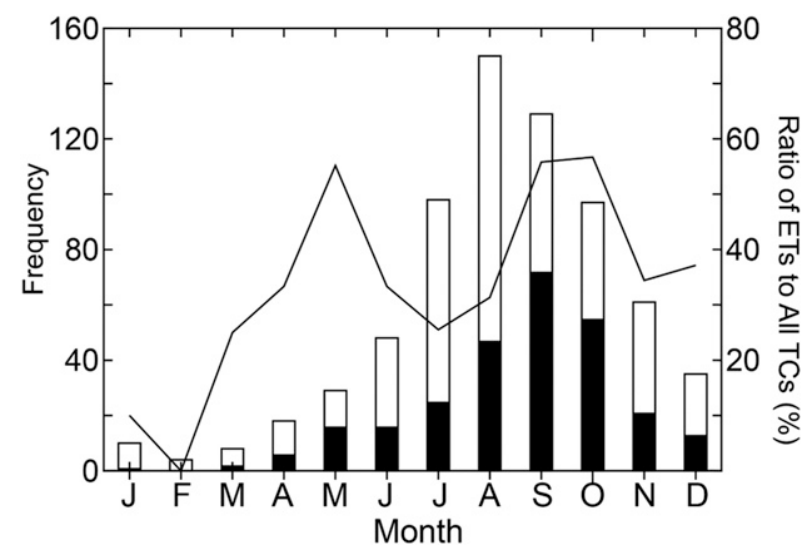

FIG. 4. Number of TCs (white bars) and number of ET cases (black bars) by month (each per left axis) during 1979-2004 in the WNP, as assessed using Japan Meteorological Agency best track data. The black line indicates the percentage of TCs that undergo ET to the total number of TCs in a given month (per right axis). Figure reproduced from Kitabatake (2011, their Fig. 10a).

States (Corbosiero et al. 2009; Ritchie et al. 2011; Wood and Ritchie 2013).

Initial case studies of ENP ET events (Dickinson et al. 2004; Corbosiero et al. 2009; Wood and Ritchie 2012) motivated the development of an ET climatology for the ENP. Because of the aforementioned negative factors of a strong ridge and poleward decreasing SSTs, only $9 \%$ of ENP TCs complete ET according to the CPS (Wood and Ritchie 2014a), a much lower frequency than in the NATL or WNP (Fig. 2). ENP ET cases require a southward-digging midlatitude trough to weaken the subtropical ridge and allow the TC to move poleward. However, when an ENP TC completes ET, it does not follow the traditional CPS path found in the WNP (Kitabatake 2011; Song et al. 2011) and NATL (Evans and Hart 2003; Arnott et al. 2004). Instead of losing thermal symmetry but retaining a warm core prior to completing ET, ENP TCs first lose their warm core and then become asymmetric as they complete ET (Fig. 5; Wood and Ritchie 2014a). Rapidly decreasing SSTs may contribute to this type of structural evolution as the loss of convection outpaces changes in the TC's thermal asymmetry, a hypothesis that is currently being investigated. Warm-core erosion prior to asymmetry development during ET is not limited to the ENP; Kitabatake (2011) found $16.8 \%$ of WNP ET cases followed this alternative CPS path. After an ENP TC completes ET, it typically decays without reintensification (Wood and Ritchie 2014a).

Similar to other basins, peak ENP ET activity occurs in September and October, which is offset from peak TC activity in July and August due to more frequent midlatitude troughs and a weaker subtropical ridge later
(Wood and Ritchie 2014a). ENP ET frequency increases during developing warm-phase El Niño-Southern Oscillation (ENSO) events, as comparatively high subtropical SST and more midlatitude troughs during warm-phase ENSO events may increase the likelihood of a TC being maintained as it moves northward and of that TC subsequently interacting with a trough (Wood and Ritchie 2014a). The ET frequency, however, appears unaffected by the longer-duration Pacific decadal oscillation (PDO; Mantua et al. 1997), possibly due to the weaker PDO influence on subtropical SST than ENSO and, thus, on TC maintenance as these systems gain latitude (Wood and Ritchie 2014a).

\section{d. Southwest Indian}

As in the ENP, an SWIO (west of $90^{\circ} \mathrm{E}$ and south of the equator) ET climatology did not exist when Jones et al. (2003) was published. The recently developed first SWIO ET climatology indicates that nearly $44 \%$ of TCs underwent ET during the 1989-2013 SWIO TC seasons ${ }^{1}$ (Fig. 2d; Griffin and Bosart 2014), rivaling the frequency of ET in the NATL (Jones et al. 2003) and exceeding that of the WNP (Kitabatake 2011; Archambault et al. 2013) and the southwest Pacific (32\%; Sinclair 2002). Whereas few ET events are observed early in the SWIO TC season (e.g., prior to January), an average of just under $50 \%$ of TCs undergo ET from January to March during the seasonal peak (Fig. 6; Griffin and Bosart 2014). This agrees with the late-season ET bias in the NATL (Hart and Evans 2001), but is a higher frequency than the late-season ET rates of $25 \%-30 \%$ in the southwest Pacific (Sinclair 2002). Further work is needed, however, to determine if this result has similar causes to the late-season ET distributions found in the WNP and NATL.

\section{e. Future climatology}

Whereas the climatologies in previous subsections have been backward looking, several studies have used observations and climate model outputs to determine how ET climatologies are currently changing or are likely to change in the future. Near Australia, increased poleward persistence of recurving TCs may result from increased higher-latitude SST and strengthened

\footnotetext{
${ }^{1}$ Depending on the data source, each Southern Hemisphere TC season begins on either 1 July (e.g., U.S. Navy Joint Typhoon Warning Center) or 1 August (e.g., World Meteorological Organization Regional Specialized Meteorological Center La Réunion) and runs for 12 months up until the beginning of the following season. Each TC season refers to the year in which the season ends, as the majority of TC events in a season occur, on average, after 1 January. For example, the 2003-04 Southern Hemisphere TC season is referred to as the 2004 season.
} 


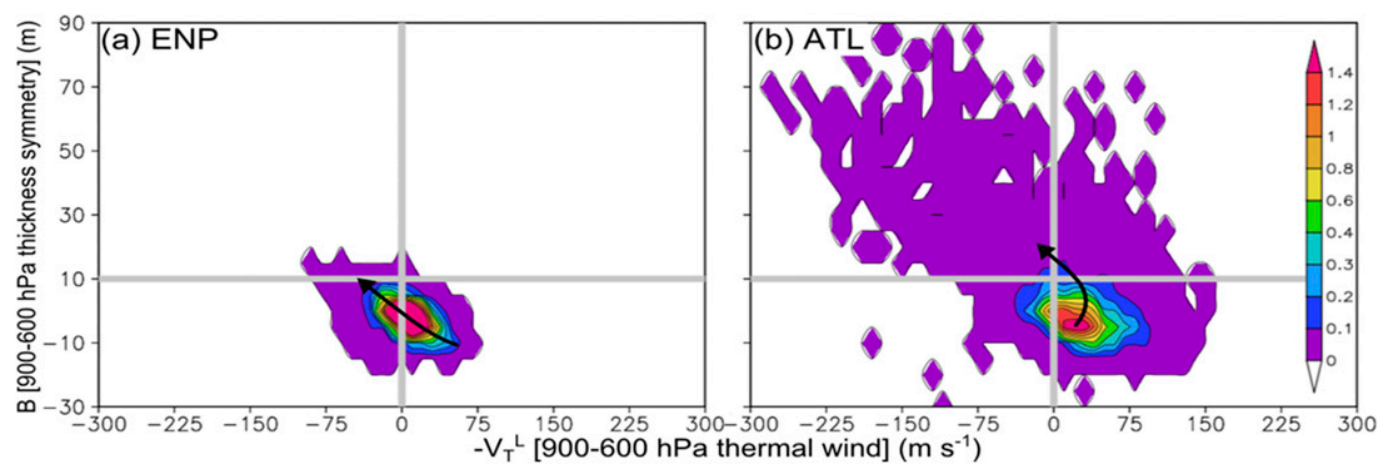

FIG. 5. Average JRA-55 CPS frequency (e.g., number of times during its life span that a given TC is located at a given location within the CPS, $-V_{T}^{L}$ and $B$ only; shaded) per TC during 2001-10 in the (a) ENP (150 TCs) and (b) North Atlantic (here, ATL; $174 \mathrm{TCs}$ ). The arrows in each panel indicate the general trajectories that TCs in each basin follow through the CPS. Figure reproduced from Wood and Ritchie (2014a, their Figs. 10a,b).

poleward-directed steering winds (Walsh and Katzfey 2000). Conversely, WNP ET likelihood may decrease as the magnitude of the meridional temperature gradient decreases (Ito et al. 2016). In the NATL, ET frequency increased over the period 1970-2012 (Mokhov et al. 2014), and several studies (Semmler et al. 2008; Haarsma et al. 2013; Baatsen et al. 2015; Liu et al. 2017) suggest further increases in NATL ET frequency in the future, particularly in the eastern portion of the basin. These projected increases were attributed to a poleward expansion of the conditions that support TCs (viz., sufficiently high SST and low deep-layer vertical wind shear) toward latitudes supportive of extratropical cyclone maintenance or intensification. Vortex interactions such as what occurred with Sandy (2012) may become less frequent if midlatitude blocking and cyclonic wave breaking frequencies decrease (Barnes et al. 2013), although there is disagreement on whether midlatitude blocking frequency will decrease in future climates (e.g., Coumou et al. 2014; Francis and Vavrus 2015).

\section{Direct impacts}

Though climatological studies can help explain the overall characteristics of ET for a given basin, whether in the past, present, or future, the impacts of an individual ET event on lives and property are directly tied to the hazards of strong winds, large waves, and heavy precipitation. During ET, the TC wind field expands and becomes increasingly asymmetric, shifting the coverage and location of maximum wind speeds and thus the regions at risk. The evolving wind field affects the distribution of large waves, and these large waves can directly impact marine interests and coastlines. In addition, extreme inland precipitation can occur within ET systems, sometimes far removed from the cyclone center. Like the wind field, the precipitation distribution tends to shift during the ET process. Jones et al. (2003) emphasized the importance of better understanding the evolution and prediction of these hazards to mitigate the societal impacts of a given ET event. Many studies have responded to these research needs since Jones et al. (2003) was published, and the following subsections
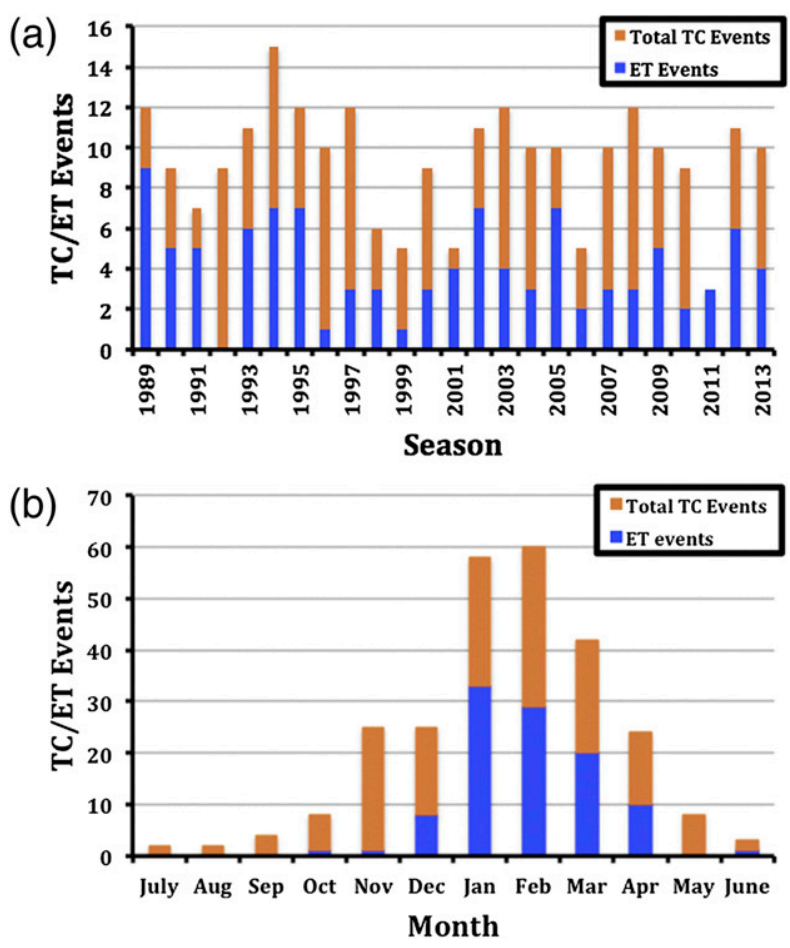

FIG. 6. Summary of TC and ET events in the SWIO west of $90^{\circ} \mathrm{E}$ by (a) TC season and (b) month for the period 1989-2013. The full height of the bar represents TC events, while the bottom (blue) portion of the bar represents the number of ET events. In (a), the year on the chart refers to the year the TC season ended. In (b), events that occur in two months are included in the month in which the TC dissipated or underwent ET. Figure reproduced from Griffin and Bosart (2014, their Fig. 1). 
examine research progress on each of the three major direct ET hazards.

\section{a. Wind}

As the TC near-surface wind field becomes increasingly asymmetric during ET (e.g., Powell 1982; Merrill 1993), with the strongest winds preferentially found to the right of track (e.g., aligned with its motion), the radius of maximum wind (RMW; the distance from the cyclone's center at which the fastest tangential wind speeds are found) moves away from the center. At the same time, winds outside the RMW increase, flattening the azimuthally averaged tangential wind profile of the cyclone and increasing the cyclone's integrated kinetic energy (Kozar and Misra 2014). As a result, the wind field expands, as illustrated by Evans and Hart (2008) through numerical simulations of NATL Hurricane Bonnie (1998). The wind field expansion has been ascribed to the following: 1 ) the import of absolute angular momentum into the cyclone as asymmetry increases, accelerating the wind field beyond the RMW; and 2) temporally and vertically integrated cooling maximized inside the RMW, weakening the radial geopotential height gradient near the center and causing the RMW to move radially outward (Evans and Hart 2008; Fig. 7). The diabatically driven expansion of cyclonic PV associated with TC wind field growth (Hill and Lackmann 2009) or sting jet development along the trailing end of a bent-back front (e.g., Browning 2004) may also contribute to the wind field evolution during ET, although further research is necessary to test these hypotheses.

Though the wind field expansion is generally associated with strong near-surface winds becoming isolated equatorward (or, for the Northern Hemisphere, right) of the TC's track during ET (e.g., Fujibe and Kitabatake 2007), in some cases strong winds can also be observed poleward (or, for the Northern Hemisphere, left) of the TC's track during ET (e.g., Fig. 8; Fujibe et al. 2006; Fujibe and Kitabatake 2007; Kitabatake and Fujibe 2009; Loridan et al. 2014). There exists disagreement, however, as to the frequency of such occurrences, with estimates ranging from $11.4 \%$ (Fujibe and Kitabatake 2007; Kitabatake and Fujibe 2009) to 66.7\% (Loridan et al. 2014) of WNP TCs near Japan. Potential causes of strong left-of-track winds during ET include frontogenesis along a warm front rearward of the TC (e.g., Riemer et al. 2006), terrain-induced flow channeling (e.g., Mashiko 2008), and the vortex response to sufficiently large vertical wind shear (e.g., Uhlhorn et al. 2014).

Knowledge of the diverse wind field structures that may arise during ET, even if only incomplete understanding exists of how and why they arise, is necessary to improve parametric wind models that can be used as input for

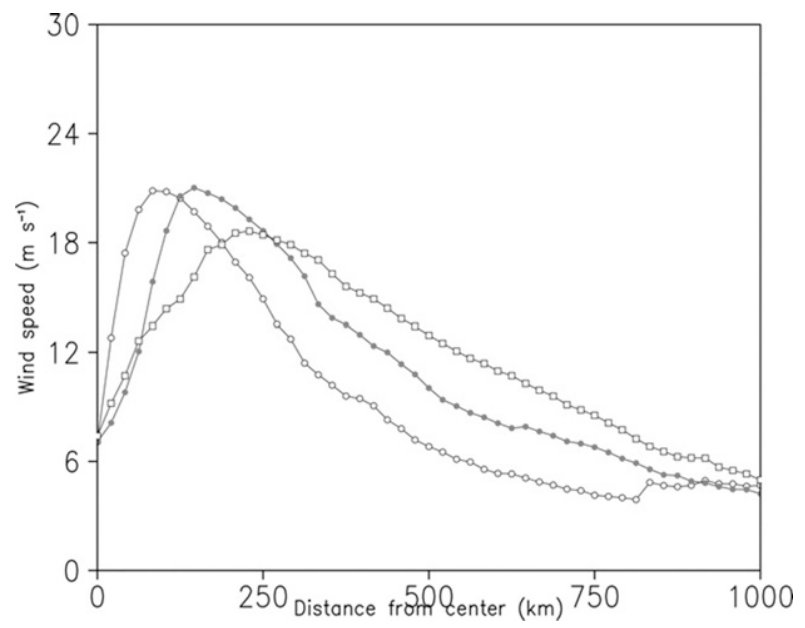

FIG. 7. Azimuthally averaged $10-\mathrm{m}$ wind speed $\left(\mathrm{m} \mathrm{s}^{-1}\right)$ as a function of radius at 0400 UTC 29 Aug (open circles; before ET), 1000 UTC 30 Aug (closed circles; during ET), and 1000 UTC 31 Aug (open squares; after ET) 1998, as obtained from the 12-km fifth-generation Pennsylvania State University-NCAR Mesoscale Model (Dudhia 1993), simulation of NATL TC Bonnie (1998). Figure reproduced from Evans and Hart (2008, their Fig. 5).

trapped-fetch wave models (MacAfee and Pearson 2006; Bruneau et al. 2017) and catastrophe models (e.g., Loridan et al. 2014, 2015). Parametric wind models typically assume strongest winds right of track, whereas there is a diverse range of near-surface wind fields that are observed during ET. Thus, to improve model skill, adjustments must be made to better represent the diversity of wind field structures observed during ET. This has been done by applying size, shape, storm motion, static stability, and/or bias-correction factors to parametric wind models commonly used at low latitudes (MacAfee and Pearson 2006; Loridan et al. 2015). In the Loridan et al. (2015) formulation, specific term values vary between transitioning TCs with a right-of-track surface wind speed maximum, left-of-track surface wind speed maximum, and right-of-track surface wind speed maximum with small cross-track asymmetry. Applying the Loridan et al. (2015) parametric wind model to storm surge prediction for idealized ET events near Japan resulted in improved storm surge and wave predictions relative to those derived using winds from a purely TC wind model (Bruneau et al. 2017).

\section{b. Waves}

The evolving TC wind field during ET has a direct influence upon the cyclone's ocean-wave field. Large ocean waves in TCs and extratropical cyclones alike pose hazards to marine activities including oil and gas extraction, fisheries, recreation, and transport. Generally, ocean wave growth is tied to cyclone characteristics such as translation speed, wind speed, and trapped-fetch length (Bowyer and MacAfee 2005; MacAfee and 
(a)

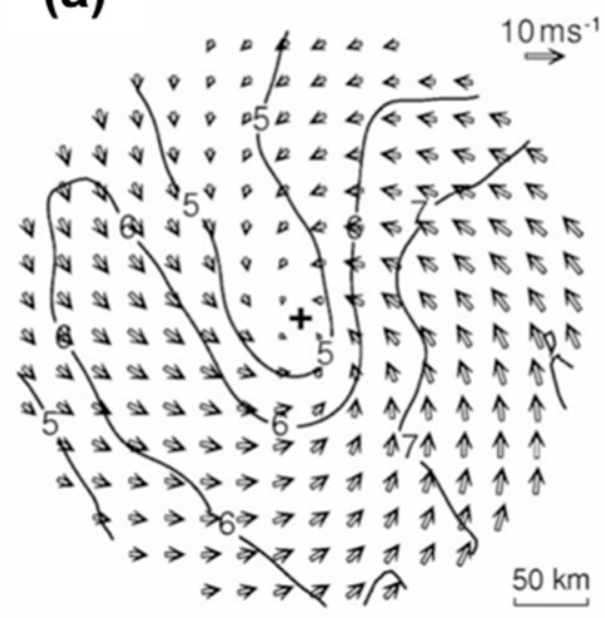

(b)

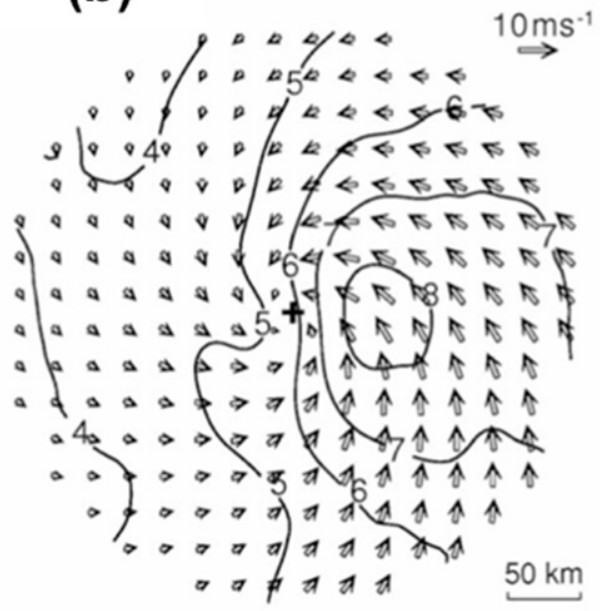

FIG. 8. Composite surface wind vectors (arrows, reference vector in the top right of each panel) and surface wind speed (isotachs, $\left.\mathrm{m} \mathrm{s}^{-1}\right)$ for (a) the subset of TCs $(n=13)$ with wind maxima both left and right of track that made landfall in Japan from 1979 to 2004 and (b) all TCs $(n=70)$ that made landfall in Japan from 1979 to 2004. The $y$ axis is taken in the direction of the storm motion. The cross in the center of each panel indicates the storm center. Figure reproduced from Fujibe and Kitabatake (2007, their Figs. 3d,f).

Bowyer 2005). Strongly enhanced waves are more likely for strong storms moving quickly with relatively small trapped-fetch lengths, with transitioning TCs having the greatest potential for these enhanced waves (Bowyer and MacAfee 2005).

Waves are small-scale phenomena, however, and their impacts tend to be parameterized in mesoscale models (if represented at all), complicating our ability to analyze and predict these features for ET events. Consequently, in addition to requiring improved inputs from meteorological forecasts, improved wave forecasts require state-of-the-art wave physics that include accurate parameterizations of the physical links between the atmosphere and ocean that govern wave behavior.

One such physical link is the generation of sea spray, which is known to contribute to TC intensification by enhancing surface latent heat flux through evaporation (e.g., Ma et al. 2015). Simulations of two TCs after ET and one of an intense extratropical cyclone demonstrated that including a sea spray parameterization increased extratropical cyclone intensity by $5-10 \mathrm{kt}(1 \mathrm{kt}=$ $0.5144 \mathrm{~m} \mathrm{~s}^{-1}$ ), depending on the storm, by increasing latent and sensible heat flux at the ocean surface (Perrie et al. 2005). The parameterization improved forecast wind speed estimates compared to those estimated from numerical model outputs and satellite observations. Wind speed, storm size, SST, and cyclone translation speed all affected the impacts of sea spray. Further research is necessary, however, to understand how sea spray generation evolves during ET (i.e., not just for TCs and extratropical cyclones separately).
Another physical link is wave drag, or the amount of friction generated by the production of ocean waves by the wind field. Using a coupled atmosphere-wave-sea spray model to investigate the combined effects of wave generation and sea spray on extratropical cyclone development, Zhang et al. (2006) found that simulated cyclone intensities better resembled their observed counterparts when including wave drag and sea spray compared to simulations without either. The influence of sea spray decreased due to decreasing SSTs as storms moved poleward, which coincided with an increasing influence of wave drag. As with sea spray, however, further research is necessary to understand how wave drag evolves during, and not just after, ET.

\section{c. Precipitation}

Although distinct from a transitioning TC's wind and wave field evolutions, precipitation is perhaps the most notable ET impact to inland locations. Indeed, precipitation from TCs that undergo ET is a partial contributor to the overall precipitation climatologies of locations such as the southeastern United States (Brun and Barros 2014; Mahoney et al. 2016) and northwestern Australia ( $\mathrm{Ng}$ et al. 2015). Rainfall associated with transitioning TCs can be directly associated with the cyclone itself or well removed (e.g., by $>1000 \mathrm{~km}$ ) from the cyclone. Although these latter events, known as predecessor rain events (e.g., Galarneau et al. 2010), can result in damaging flash floods, they are considered an indirect impact of ET and are discussed in more detail in Part II. Several heavy-precipitation events associated 
with transitioning TCs have caused historic flash floods in the United States well inland from the Atlantic coast, including Hazel in 1954 (Palmén 1958; Matano 1958), Agnes in 1972 (e.g., Carr and Bosart 1978; Bosart and Dean 1991), and Irene in 2011 (e.g., Milrad et al. 2013; Smith et al. 2016).

Because heavy precipitation associated with ET can occur well inland, as the cases cited above indicate, it is crucial to understand where heavy precipitation occurs during ET and the conditions that cause ET-related heavy-precipitation events to inform timely warnings. During ET, precipitation shifts radially outward and has maximum intensity downshear (Matyas 2010a,b,c). Precipitation coverage grows in areal extent as ET begins, but decreases in areal extent later in the process (Matyas 2013). Whereas TCs are typically characterized by heaviest precipitation to the left of the vertical wind shear (e.g., Lonfat et al. 2004; Chen et al. 2006), which is often right of track in the NATL, the heaviest precipitation during ET may be found either left or right of track (Atallah and Bosart 2003; Atallah et al. 2007; Milrad et al. 2009; Chen 2011; Zhou and Matyas 2017). Left-of-track precipitation is more common under atmospheric conditions resembling those favoring reintensification after becoming extratropical (section 5b), notably a negative-tilted upstream trough near to, and of similar scale as, the TC, with amplified mid- to uppertropospheric ridging atop and downstream of the TC (Atallah et al. 2007; Milrad et al. 2009). Conversely, cases with right-of-track precipitation maxima generally never completed ET or decayed shortly after becoming extratropical. Despite advanced understanding of precipitation field evolution during ET, however, the extent to which precipitation asymmetries evolve and/or are in phase with the wind, wave, and thermal asymmetries warrants further study.

Though orography has long been known to affect TC precipitation rates, particularly in Taiwan (e.g., Lin et al. 2001; Yu and Cheng 2008, 2013), it may also focus extreme precipitation during ET. For example, Vermont was heavily impacted by flash flooding from NATL TC Irene (2011), largely due to orographic precipitation enhancement in the complex terrain of the Green Mountains (Liu and Smith 2016; Smith et al. 2016). Similarly, as precipitation with NATL TC Sandy (2012) shifted to the left of track during and after ET, upslope flow induced by the cyclone within a highly anomalous antecedent cold air mass along the western slopes of the Appalachian Mountains of North Carolina, Virginia, and West Virginia resulted in up to $900 \mathrm{~mm}$ of snowfall (Keighton et al. 2016). Only three prior NATL TCs are known to have produced accumulating snow in the United States, all of which occurred in New England in fall or winter in the presence of elevated terrain and an antecedent cold air mass (Keighton et al. 2016). Orography can also modulate a transitioning TC's rainfall distribution, as has been seen with systems in the St. Lawrence River valley of Canada (Milrad et al. 2009, 2013). Here, ageostrophic frontogenesis focused by the local topography, rather than the synoptic-scale pattern, has been found to exert primary control on where the heaviest precipitation occurs with transitioning TCs in this region (Milrad et al. 2013).

\section{Structural evolution}

The potential impacts of winds, waves, and precipitation are tied to the evolving structure of the transitioning cyclone. Historically, numerical model simulations and model-derived observation syntheses have been the primary means of obtaining insight into cyclone structure evolution during ET (Jones et al. 2003). This prompted Jones et al. (2003) to stress the critical need for increased in situ observations and targeted field experiments of ET events to validate the insights gleaned from numerical simulations and to improve our knowledge of the ET process.

In recent years, several field experiments with partial ET foci have been conducted. NOAA's Intensity Forecasting Experiment (IFEX; Rogers et al. 2006, 2013) included flights into NATL TC Ophelia (2005) that provided novel observations of a transitioning TC's structural evolution using airborne Doppler radar and dropsondes (Fogarty 2006; Rogers et al. 2006). Later IFEX missions sampled Earl (2010), Sandy (2012), Arthur (2014), Kate (2015), and Karl (2016) before and during ET, including Sandy's interrupted transition east of Florida (Blake et al. 2013). Additionally, three separate programs investigated WNP ET during 2008: The Observing System Research and Predictability Experiment (THORPEX)-Pacific Asian Regional Campaign (T-PARC; Elsberry and Harr 2008; Waliser et al. 2012), the Dropsonde Observations for Typhoon Surveillance near the Taiwan Region (DOTSTAR; Wu et al. 2005) experiment, and the Tropical Cyclone Structure (TCS-08; Elsberry and Harr 2008) experiment.

Together, observation- and numerical model-based studies have advanced understanding of structural evolution both during and after ET. In particular, observations have provided validation of previously developed conceptual models, and numerical model-based studies have increased our knowledge of variation and environmental sensitivities in structural evolution during and after ET. Organized around the two-stage ET conceptual model highlighted in Fig. 1 and the three-step conceptualization of the transformation stage of Klein et al. (2000; 
(a) UL
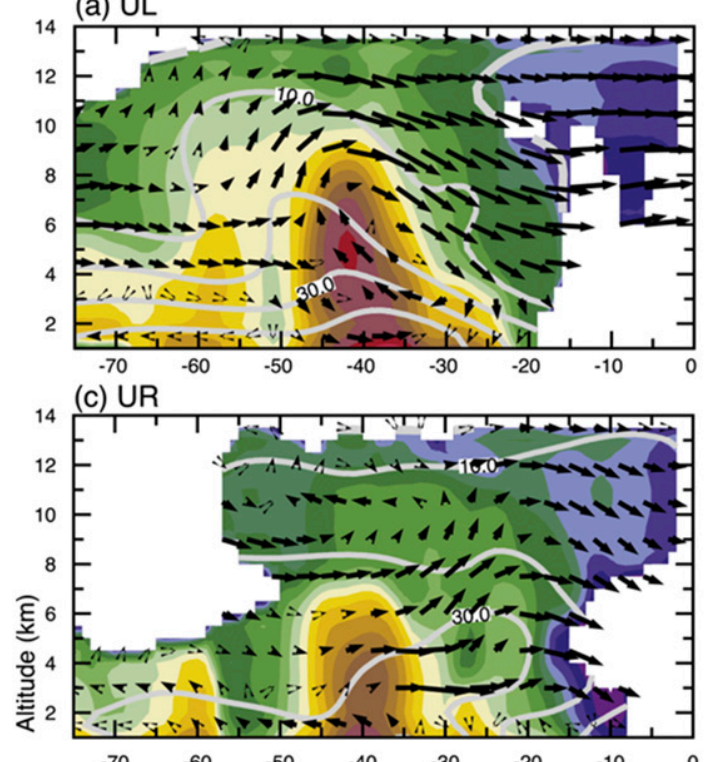

$-70 \quad-60$

Radius(km) (b) DL

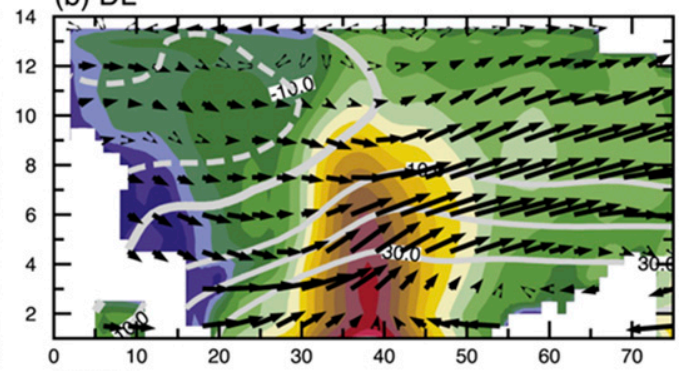

(d) DR

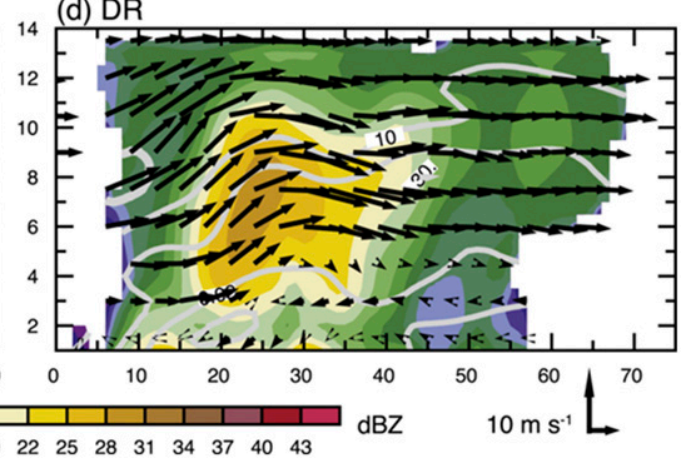

FIG. 9. Vertical cross sections of radar reflectivity (dBZ; shaded), tangential wind ( $\mathrm{m} \mathrm{s}^{-1}$; gray contours), and inplane wind vectors composed of radial and vertical velocity $\left(\mathrm{m} \mathrm{s}^{-1}\right)$ for each shear-relative quadrant as synthesized by the Spline Analysis at Mesoscale Utilizing Radar and Aircraft Instrumentation (SAMURAI; Bell et al. 2012) software tool for the T-PARC research mission into Typhoon Sinlaku on 19 Sep 2008. Cross sections are taken from the corner of the domain to the center, $45^{\circ}$ from the $x$ and $y$ axes in each quadrant: (a) upshear left, (b) downshear left, (c) upshear right, and (d) downshear right. Figure reproduced from Foerster et al. (2014, their Fig. 12).

Fig. 3), this section discusses insights gained from observations as well as numerical simulations and analyses. The extensively studied case of WNP Typhoon Sinlaku (2008) is used as a representative example of structural evolution during the transformation stage.

\section{a. Transformation stage}

\section{1) STEP 1: INITIAL ENCOUNTER WITH THE MIDLATITUDE BAROCLINIC ZONE}

Step 1 is characterized by the initial encounter of a TC's outer circulation with a midlatitude baroclinic zone and its translation poleward over lowering SSTs (Klein et al. 2000). During this process, idealized model simulations suggest that TCs weaken as they encounter decreasing SSTs and increasing vertical wind shear magnitude (Ritchie and Elsberry 2001). As the cyclone weakens, deep, moist convection and associated precipitation become more asymmetric, the wind field expands (section 4a), and the TC warm core weakens.

In the case of Typhoon Sinlaku (2008), observations suggest inner-core asymmetries during this step most likely resulted from strong southwesterly deep-layer vertical wind shear impinging upon the TC (Foerster et al. 2014). Convective cells tended to form downshear right (Fig. 9d), advect cyclonically and reach maximum intensity downshear left (Fig. 9b), and decay upshear (Figs. 9a,c; Foerster et al. 2014). This pattern is consistent with previous studies of mature TCs in vertical wind shear (e.g., Marks et al. 1992; Frank and Ritchie 2001; Black et al. 2002; Corbosiero and Molinari 2002; Reasor et al. 2009; DeHart et al. 2014). The exposure to strong vertical wind shear contrasts with the identical response seen in the simulations of Ritchie and Elsberry (2001), potentially because of timing differences within the first step of transformation between the two studies.

\section{2) Step 2: Superposition With THE MIDLATITUDE BAROCLINIC ZONE}

During step 2, the cyclone becomes nearly collocated with the midlatitude baroclinic zone, begins to acquire substantial vertical tilt, and develops structures such as conveyor belts (Carlson 1980; Browning 1990, 2004) characteristic of extratropical cyclones (Klein et al. 2000). During this process, deep, moist convection becomes constrained to the northwest quadrant with strong ascent in the eyewall and a thin cirrus shield to the north and northeast (Klein et al. 2000; Ritchie and Elsberry 2001). Aircraft observations acquired in NATL TCs Michael and Karen in 2000 (Wolde et al. 2001; Abraham 
et al. 2004) and Ophelia in 2005 (Fogarty 2006; Rogers et al. 2006) provided in situ observations corroborating this step of the Klein et al. (2000) conceptual model.

Despite its contribution to increased vortex tilt, moderate-to-strong vertical wind shear encountered during this step can increase the area-averaged vertical mass flux, potentially to values exceeding those found in the mature TC prior to the transformation stage (Davis et al. 2008). This increased vertical mass flux may result from quasi-balanced vertical motion superposed on the TC secondary circulation, leading to saturation and invigorated convection downshear but suppressed convection upshear. An optimal 1-7-km vertical wind shear magnitude of $15 \mathrm{~m} \mathrm{~s}^{-1}$ was found to maximize total vertical mass flux during ET, implying the vortex can resist moderate vertical wind shear magnitudes through intense diabatic heating caused by deep, moist convection (Davis et al. 2008).

For Typhoon Sinlaku, observations indicate that the primary cause of asymmetric structure shifted during this step from a response to increased vertical wind shear to processes associated with the vortex's superposition with a midlatitude baroclinic zone. During this step, Sinlaku was characterized by reduced convection west, but deep, moist convection east of the center (Quinting et al. 2014). Deep, moist convection developed in response to meso- to synoptic-scale forcing for ascent where the leading edge of the dry-air intrusion from the west intersected an intensifying warm front (Fig. 10). A similar evolution was also observed in WNP Typhoon Phanfone (2014) by polarimetric radar observations collected on board the Research Vessel (R/V) Mirai (Katsumata et al. 2016). Together, these studies provide observational validation of Klein et al. (2000) and Ritchie and Elsberry (2001), particularly with respect to the increasingly dominant role of the midlatitude baroclinic zone in fostering asymmetric structural evolution as transformation proceeds.

\section{3) STEP 3: FinAl DEVELOPMENT OF EXTRATROPICAL STRUCTURE}

During step 3, the structural evolution that began in step 2 completes as the TC becomes fully embedded within the midlatitude baroclinic zone and loses its tropical structure (Klein et al. 2000). The idealized modeling study of Ritchie and Elsberry (2001) indicated the TC's cyclonic circulation was contained below $400 \mathrm{hPa}$, with maximum intensity located near $750 \mathrm{hPa}$ and a notable northeast vertical tilt at higher altitudes, during this step. A secondary warm anomaly adjacent to the southwest eyewall, also observed in Sinlaku by Quinting et al. (2014) during step 2, was reinforced by strong subsidence associated with the descending dry

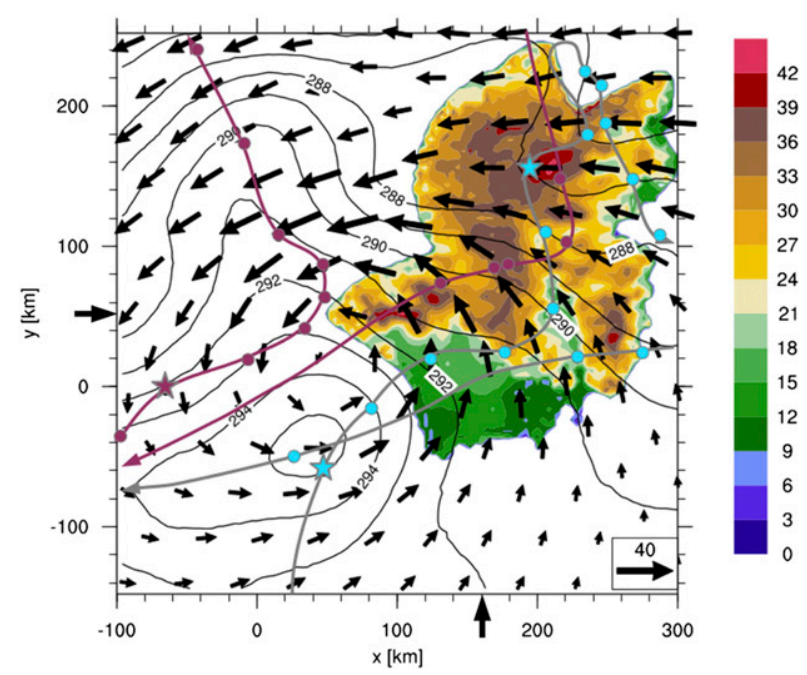

FIG. 10. Maximum reflectivity between 0 and $15 \mathrm{~km}(\mathrm{~dB} Z$; shaded), temperature ( $\mathrm{K}$; contours) at $1.5 \mathrm{~km}$, and horizontal wind $\left(\mathrm{m} \mathrm{s}^{-1}\right.$; vectors with reference vector at lower right) at $1.5 \mathrm{~km}$ as synthesized by SAMURAI for the T-PARC research mission into Typhoon Sinlaku on 20 Sep 2008. The gray line denotes the flight track of the NRL-P3 and the red line denotes the flight track of the USAF-WC130. Filled circles give positions of dropsondes included in the SAMURAI analysis. Stars indicate positions of dropsondes in Fig. 7 in Quinting et al. (2014), while black arrows along the coordinate axes indicate positions of cross sections in Fig. 6 in Quinting et al. (2014). Figure reproduced from Quinting et al. (2014, their Fig. 4).

intrusion and was associated with a weak sea level pressure depression. The southwest quadrant was completely dry due in part to both subsidence-induced drying along the descending dry intrusion airstream and the TC-induced advection of cold, dry midlatitude air. Whereas the precipitation maximum was left of track, consistent with the location of maximized cyclonic vorticity advection by the thermal wind (e.g., Atallah et al. 2007), the maximum near-surface wind speeds were right of track and aligned with the idealized TC's northeastward motion.

Although fewer observations exist during the third step of transformation, real-data numerical simulations of Sandy (2012) and Sinlaku (2008) have documented variability in the archetypical structural evolution as transformation completes. In Sandy, the TC's warm core was surrounded by cold, continental polar air as it moved northward (Fig. 11a; Galarneau et al. 2013). By the time Sandy completed ET, the $850-\mathrm{hPa}$ thermal structure (Fig. 11b) resembled that of a warm-seclusion cyclone (Shapiro and Keyser 1990), which occurs in approximately one-third of ET events (Kitabatake 2008). Vortex intensification in an environment of increased baroclinicity during ET was driven primarily by enhanced low-level convergence that resulted in 
a) AHW 34-h Forecast (10Z/29)

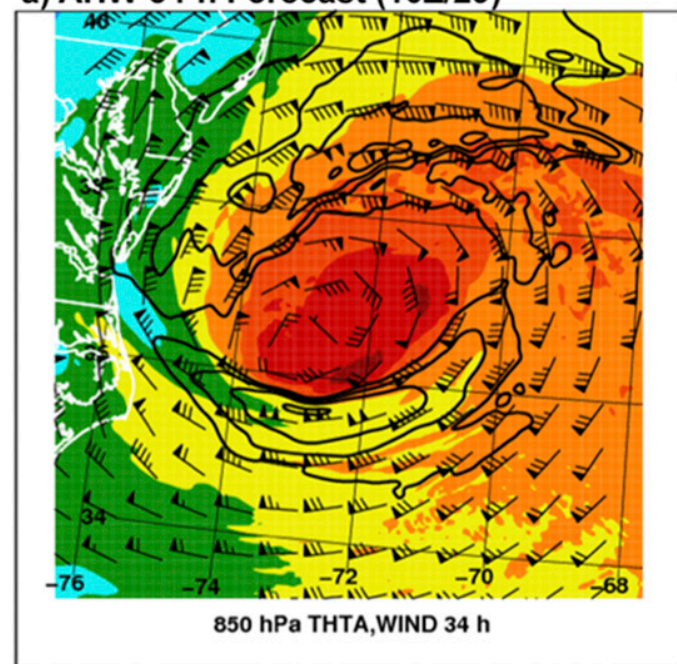

b) AHW 44-h Forecast (20Z/29)

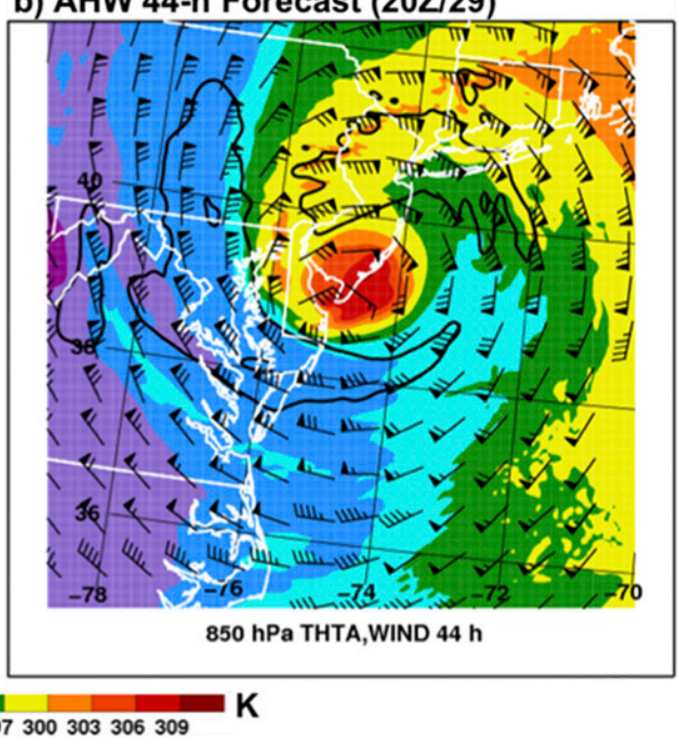

FIG. 11. Advanced Hurricane Weather Research and Forecasting (AHW; Davis et al. 2010) model-forecast 850-hPa potential temperature $\left(\mathrm{K}\right.$; shaded), vector wind (half barb $=2.5 \mathrm{~m} \mathrm{~s}^{-1}$; full barb $=5.0 \mathrm{~m} \mathrm{~s}^{-1}$; pennant $=$ $25.0 \mathrm{~m} \mathrm{~s}^{-1}$ ), and wind speed (solid contours at 40, 45, 50, and $55 \mathrm{~m} \mathrm{~s}^{-1}$ ) of Sandy (2012) verifying at (a) 1000 and (b) 2000 UTC 29 Oct 2012. The AHW forecast was initialized at 0000 UTC 28 Oct 2012. Figure reproduced from Galarneau et al. (2013, their Figs. 7b,c).

cyclonic vorticity amplification via vortex tube stretching (Galarneau et al. 2013), lower-stratospheric warming (Shin and Zhang 2017), and latent heat release within a partial remnant eyewall and along an inwardcontracting frontogenesis-driven rainband (Shin and Zhang 2017).

In Sinlaku (Lentink 2017), the prevailing lowertropospheric flow to the north was blocked from entering Sinlaku by mountains across Japan (Fig. 12a) as the cyclone approached Japan from the south. The mountains protected Sinlaku from entraining cold, dry, midlatitude air located to its northwest while also permitting its tropical reintensification. Tropical reintensification abruptly ended $6 \mathrm{~h}$ later as local orography began to favor the cyclonic inflow of cold, dry air originating to Sinlaku's northeast (Fig. 12a). When Sinlaku moved away from Japan's orographic barrier, the cold, dry midlatitude air to the northwest was no longer inhibited from being entrained into Sinlaku's circulation (Fig. 12b). Pronounced cold and warm sectors subsequently evolved, reflecting the culmination of Sinlaku's ET, following the transformation process outlined by Klein et al. (2000) and Ritchie and Elsberry (2001).

\section{b. Extratropical stage and posttransition outcomes}

As ET concludes, the system may dissipate, merge with an existing extratropical cyclone, or reintensify (Fig. 1). These different outcomes result in a wide range of potential impacts and are associated with distinct atmospheric and oceanic environments; yet, until recently, little was known about the causes of the disparate cyclone evolutions during the extratropical stage (Jones et al. 2003).

In a composite analysis of NATL ET events, cyclones that intensified post-ET (here defined using the CPS definition described in section 2) were, on average, associated with a negative-tilted midlatitude trough axis approximately $1000 \mathrm{~km}$ upstream of the cyclone's center, whereas cyclones that weakened post-ET were associated with a positive-tilted midlatitude trough approximately $1500 \mathrm{~km}$ upstream (Hart et al. 2006). Cyclones that acquired a warm-seclusion structure postET did so when the horizontal scale of the upstream trough closely matched that of the transitioning TC, akin to the favorable trough interaction composites of Hanley et al. (2001). These distinctions were attributed to variability in the upper-tropospheric transport of positive PV by atmospheric eddies in proximity to the transitioning TC (Hart et al. 2006). Each cyclone sample considered by Hart et al. (2006) was small, however, and their study references a few specific cases (e.g., those studied by Thorncroft and Jones 2000) that do not conform to the composites. Other recent case studies of individual ET events provide both support for (e.g., McTaggart-Cowan et al. 2007; Pantillon et al. 2013), and contrasting views from (e.g., Evans and Prater-Mayes 
(a)

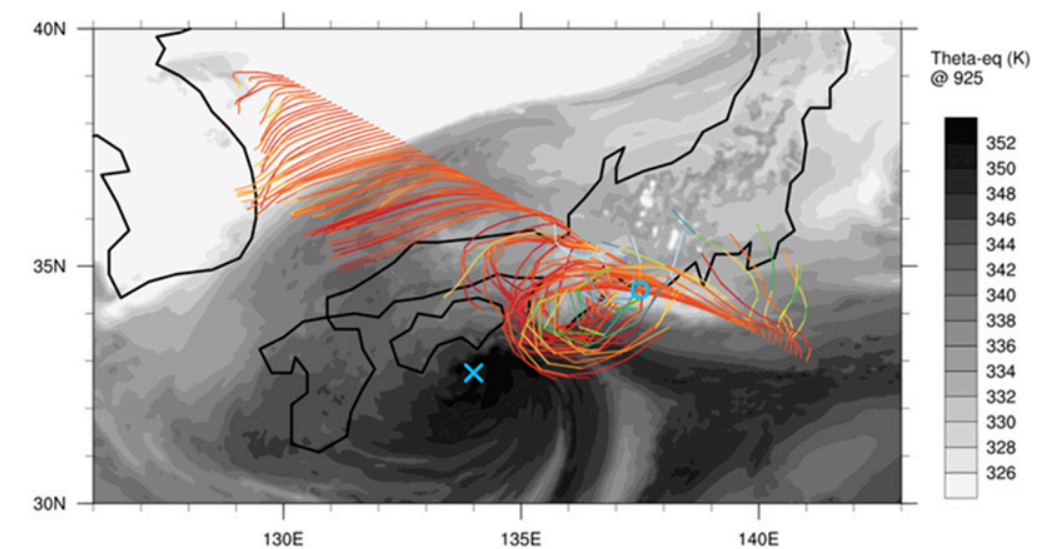

(b)

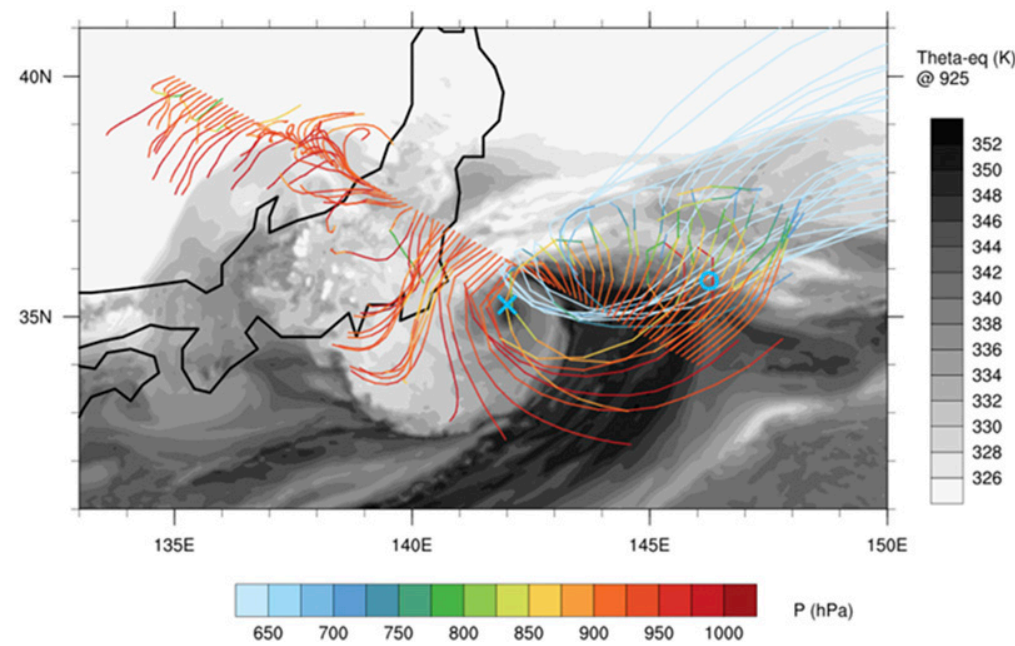

FIG. 12. 12-h forward trajectories starting at $925 \mathrm{hPa}$ on a northwest-southeast-directed line crossing Japan at (a) 0000 UTC 19 Sep 2008 and (b) 0000 UTC 20 Sep 2008. The colors of the trajectories represent pressure $(\mathrm{hPa})$. Equivalent potential temperature $(\mathrm{K})$ at $925 \mathrm{hPa}$ at trajectory starting time is given in gray shades. The location of Sinlaku's simulated mean sea level pressure minimum is marked by a blue cross (at trajectory start) and circle (at trajectory end). Figure reproduced from Lentink (2017, their Figs. 5.3 and 5.20b).

2004; Sun et al. 2012), the composite-derived insights of Hart et al. (2006).

Insight into intensity change during the extratropical stage has also been gained from idealized numerical simulations. Cyclone intensity after the transformation stage was found to be sensitive to the phasing between the posttropical cyclone and upstream trough, largely independent of the latter's strength (Ritchie and Elsberry 2003, 2007). The areal amount and amplitude of the Petterssen development parameters (Petterssen 1956) of midtropospheric cyclonic vorticity advection and lower-tropospheric temperature advection, as well as upper-tropospheric divergence (Klein et al. 2002), differed between cyclones that dissipated or reintensified immediately after the end of the transformation stage, with higher values of each parameter favoring reintensification (Ritchie and Elsberry 2007).
Proper phasing of the cyclone with the midlatitude trough, defined where the cyclone is positioned in a region favorable for extratropical cyclone development ahead of the trough (e.g., Fig. 1 of Ritchie and Elsberry 2007), also resulted in larger lower-tropospheric equivalent potential temperature air associated with the TC's remnants becoming entrained into the developing extratropical cyclone rather than when this phasing was not achieved. The lifting of this warm, moist air resulted in precipitation greater in both amount and areal extent than in the absence of phasing, enhancing extratropical development (Ritchie and Elsberry 2003).

\section{Forecasting and analysis}

Understanding the evolution of cyclone structure and associated hazards during ET is important to improve 
both forecast quality and communication. By better quantifying the dynamic controls on ET and its outcomes, forecasts of these events should become more accurate. More accurate forecasts should, in turn, result in more timely warnings that save lives and property, so long as those forecasts convey the evolving hazards (section 4) in an accessible fashion. This section discusses ET forecasting and analysis methods currently in use by operational centers, the current levels of ETrelated track and cyclone classification skill, and sensitivities inherent to track forecasts during ET and methods by which such sensitivities may be partially overcome.

\section{a. Operational applications}

Operational ET forecasting practices vary between agencies with respect to terminology, advisory, and communication practices, in part because of the differing hazards most commonly experienced in the various regions (Fogarty 2010). An overview of ET-related operational forecast practices and challenges for many World Meteorological Organization Regional Specialized Meteorological Centers and other agencies with TC forecasting responsibilities is provided by Fogarty (2010), thus only a brief summary is included here. Representing a notable ET-related research-to-operations success of the sort advocated by Jones et al. (2003), it is believed that all operational forecast agencies use the CPS and conceptual models (e.g., Fig. 3) to diagnose ET. Scatterometers (e.g., Brennan et al. 2009), microwave satellite imagery, and ship and buoy observations are used to analyze the evolving TC wind and precipitation fields as well as to validate numerical model initializations. Primary hazard warnings include wind, waves, and precipitation, with the latter a lesser emphasis for centers with lower populations in the midlatitudes (e.g., La Réunion, France). For centers such as the Japan Meteorological Agency, Canadian Hurricane Center, and National Hurricane Center (NHC), conveying the significant and evolving hazards associated with TCs during and after ET has posed a communications challenge that prompted changes to operational advisory practices.

Indeed, even a skillful ET forecast can be of limited practical use if the public and decision-makers are confused by terminology and changes in forecast responsibility between agencies, or if the public does not have an accurate perception of their individual risk associated with the event. This became acutely aware to the public in the lead-up to, and aftermath of, Sandy's 2012 landfall in New Jersey (Blake et al. 2013; Fogarty and Blake 2013). During the event, Sandy's impacts were anticipated quite accurately by NHC and National Weather
Service forecasters during both the tropical and extratropical phases of the storm. The designation of the TC as extratropical prior to landfall and the changes to advisory products that it prompted led to confusion among end users as to the cyclone's classification (NOAA 2013). Despite this, coastal residents had universally high awareness of the threats associated with Sandy's landfall, even as they had less accurate understanding of the specific hazard that posed the greatest impact to their location and its expected duration and severity (Meyer et al. 2014). Sandy prompted changes, first implemented in 2016 for TC Matthew along the U.S. East Coast, to operational NHC practices for TCs expected to become extratropical while still posing a threat to land, with the resulting "post tropical" advisory products similar in focus to those issued by the NHC during the tropical phase and to those issued by the Canadian Hurricane Center and Japan Meteorological Agency after a TC has become extratropical (Fogarty 2010). Further collaborative research between physical and social scientists, however, is necessary to improve hazard and risk communication and perception, particularly in light of the evolving threats during ET.

Another ET-related operational challenge is associated with the remote assessment of cyclone intensity during ET. It is well known by operational forecasters (e.g., at La Réunion and the Joint Typhoon Warning Center; Fogarty 2010) that the empirical relationships between cloud patterns and cyclone intensity that underlie the Dvorak technique (DT; Dvorak 1984) and advanced Dvorak technique (ADT; Olander and Velden 2007) are less reliable during ET than at other times (Velden et al. 2006). During ET, this decrease in reliability results in unrepresentative estimates of the cyclone's true intensity (Miller and Lander 1997; Knaff et al. 2010) and arises due to structural changes that lead to progressively weaker DT- and ADT-derived intensity estimates (Knaff et al. 2010), despite baroclinic energy conversion that can help maintain cyclone intensity. Consequently, in the context of the ADT, maximum wind speed estimates have large errors $(14 \mathrm{kt})$ and biases $(-13 \mathrm{kt})$ during ET that are largest when ET begins, especially in strong TCs. These errors decrease only as the transitioning TC weakens toward the lowest-possible ADT-derived intensity estimates (Manion et al. 2015). Research is ongoing to develop a bias correction for the ADT that is applicable during ET across all TC basins.

Despite the analysis, forecast, and communication challenges associated with ET, recent improvements in deterministic and ensemble numerical guidance (e.g., Aberson et al. 2015; Zhang and Weng 2015; Weng and Zhang 2016), as well as observational capabilities, have benefited operational centers. Beven (2012b) demonstrated that these improvements have contributed to 

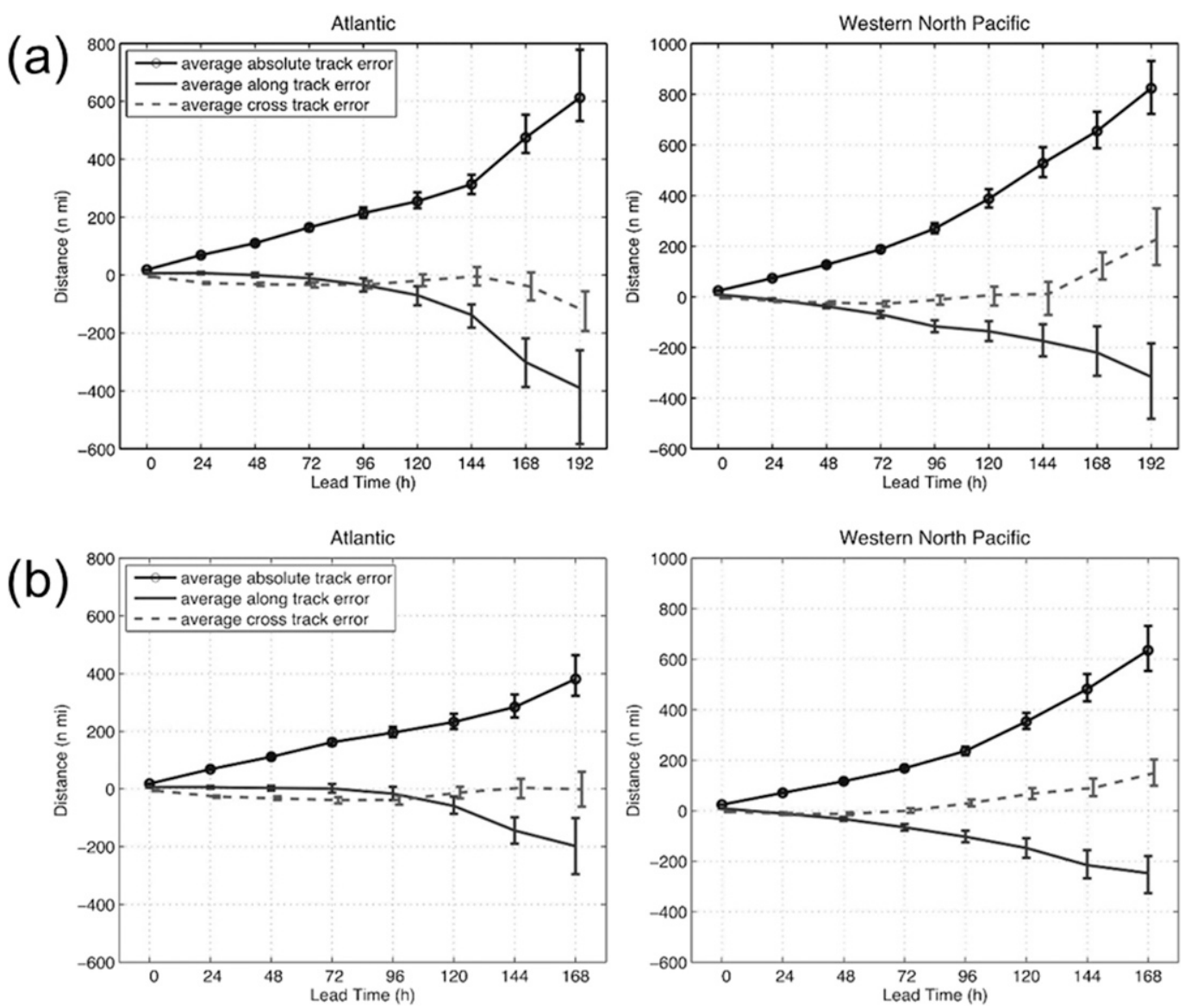

FIG. 13. The average absolute and along- and cross-track errors of the NCEP Global Ensemble Forecast System in the NATL and WNP basins for the period 2006-08. Error bars illustrate 95\% confidence intervals on the mean as determined using bootstrapping. Both TC and ET tracks are included in the analysis. Along-track error is positive when a forecast lies ahead of its verifying position and cross-track error is positive when a cyclone is forecast to the right of its verifying position. Figure reproduced from Buckingham et al. (2010, their Fig. 4). (b) As in (a), but only TC tracks are included in the analysis (ET tracks excluded). Figure reproduced from Buckingham et al. (2010, their Fig. 5).

increased skill of NHC forecasts of NATL ET timing and occurrence, although no attempt was made in their study to account for evolving ET classification practices. Advanced Microwave Sounding Unit temperature retrievals have been routinely used as CPS inputs to obtain analyses that complement those derived from numerical models, particularly at the NHC. Further, multispectral observations of moisture, temperature, and ozone concentration allowed for the creation of new imagery to improve identification of evolving cyclone structures during ET (Beven 2012a), first for Europe and Asia and more recently for North America as new geostationary satellites became operational in each region.

\section{b. Assessments of forecast skill}

An operational center's ET forecast should be assessed within the context of expected numerical model forecast skill, including insight as to when skill is likely to be either anomalously high or low. There are two ETrelated forecast elements for which extensive verification of operational and/or numerical model forecasts have been conducted: cyclone classification (e.g., tropical vs extratropical) and, to greater extent, track. For example, ET cases were associated with large along- and cross-track forecast errors at and beyond $24 \mathrm{~h}$ in the WNP and $72 \mathrm{~h}$ in the NATL in 2006-08 Global Ensemble Forecast System (GEFS; e.g., Toth and Kalnay 1997; Buizza et al. 2005) forecasts as compared to nonET TC cases (Fig. 13; Buckingham et al. 2010). These errors can result from rapid translation speeds during ET and incorrect phasing between the TC and upstream trough (e.g., Ritchie and Elsberry 2007), with subsequent impacts manifesting both locally and downstream (Part II). A representative example of this is 
given by Sandy (2012), wherein GEFS members with inaccurate track forecasts had inaccurate cyclone classification forecasts (Kowaleski and Evans 2016).

Studies have verified other ensemble forecast-system forecasts of cyclone track and classification during ET, highlighting specific situations in which forecast skill is particularly low. In the European Centre for MediumRange Weather Forecasts (ECMWF) Ensemble Prediction System (Molteni et al. 1996; Buizza et al. 2001) forecasts of two WNP ET events, error was largest for forecasts verifying during, rather than prior to, ET (Veren et al. 2009). Forecast error saturated at approximately $60 \mathrm{~h}$ for both cases and was relatively constant thereafter. For forecasts initialized at lead times of greater than $36 \mathrm{~h}$, both cases were characterized by an average delay of at least $24 \mathrm{~h}$ in predicting the time of ET transformation stage completion. Lower-tropospheric warm-core intensity was the primary factor that resulted in degraded cyclone classification forecasts, with forecasts struggling to adequately weaken the warm core during ET. In contrast, the thermal asymmetry was well predicted and, in contrast to Sandy (Kowaleski and Evans 2016), forecast-track error only minimally influenced cyclone classification forecast skill. However, over a larger sample of cases fostered by the THORPEX Interactive Grand Global Ensemble (TIGGE; Bougeault et al. 2010), only the ECMWF ensemble consistently covered the full range of possible ET scenarios (Keller et al. 2011).

In contrast to track forecasts, cyclone classification forecast skill has remained largely unknown until recently. An evaluation of numerical model cyclone classification analyses and forecasts (to $36 \mathrm{~h}$ ) for NATL TCs from 1998 to 2002 by Evans et al. (2006) found that cyclones undergoing ET, particularly near the completion of the transformation stage, were associated with the worst short-range forecast skill (of all cyclones considered) that resulted primarily from a slow bias in depicting ET completion. Vortex bogusing improved classification forecasts prior to ET, at least for relatively coarse models unable to resolve TCs, but degraded classification forecasts during ET. Operational NHC forecasts since 2000 also have a slow bias in ET completion time (Beven 2012b), which might result from a slow bias in the numerical model guidance as the TC accelerates poleward. In total, track error is a substantial contributor to cyclone classification error in some cases (e.g., Kowaleski and Evans 2016), but not in others, and further study is necessary to understand why.

More recently, a "no skill" model has been developed using linear discriminant analysis to provide a climatological baseline TC classification forecast to aid in the evaluation of operational and numerical model forecast

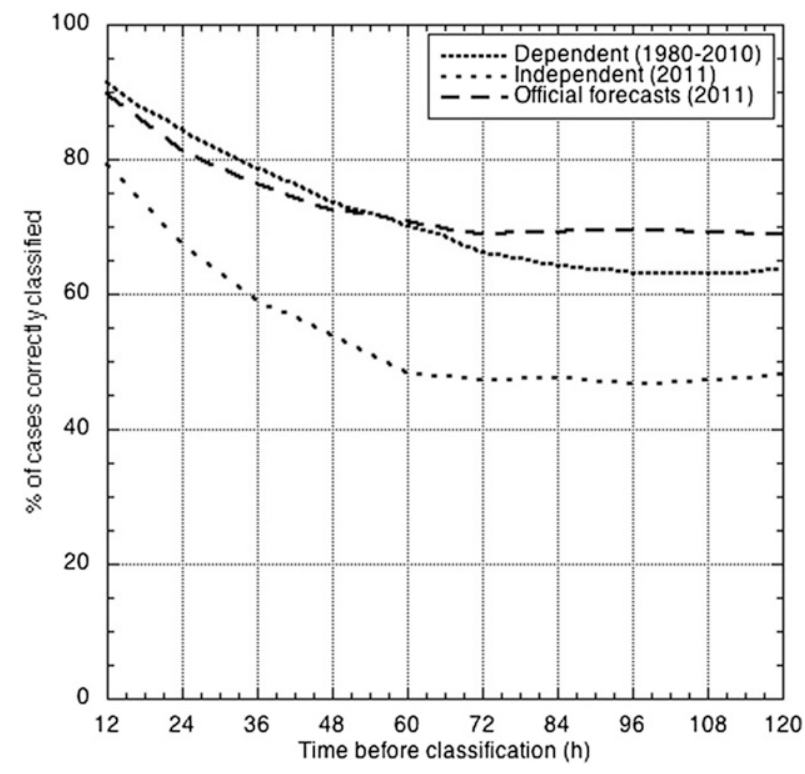

FIG. 14. Percentage of correctly classified cyclone phase forecasts by the linear discriminant analysis scheme of Aberson (2014) for dependent (short dashed; period of record 1980-2010) and independent (medium dashed; period of record 2011) samples. The long-dashed line indicates the percentage of correctly classified official NHC cyclone phase forecasts (period of record 2011). Note that the two 2011 samples are homogeneous. Figure reproduced from Aberson (2014, their Fig. 3).

skill (Aberson 2014). Using predictors that included position and intensity, the 12-h trend for each, and the current day of the year, NHC cyclone classification forecasts were found to be skillful at all lead times (Fig. 14). The small independent sample size did not permit ET forecasts to be isolated, however, and ET timing forecast skill relative to the baseline forecast remains to be quantified for both NHC and other operational center forecasts.

\section{c. Numerical model investigations of forecast sensitivity during ET}

Knowledge of scenarios associated with large forecast errors has motivated multiple in-depth investigations of the associated sensitivities and evaluations of means by which they may be ameliorated, particularly with respect to the track during ET. For example, weak initial vortex representations in short-range operational model forecasts of Juan's (2003) landfall in Nova Scotia (Fig. 15) led to reduced downstream ridge amplification and a steering flow erroneously dominated by lowertropospheric easterly winds (McTaggart-Cowan et al. 2006a). For this case, model systems with vortex initialization generally had much better success with landfall prediction, a somewhat surprising result given that the assumptions of storm symmetry that underlie 

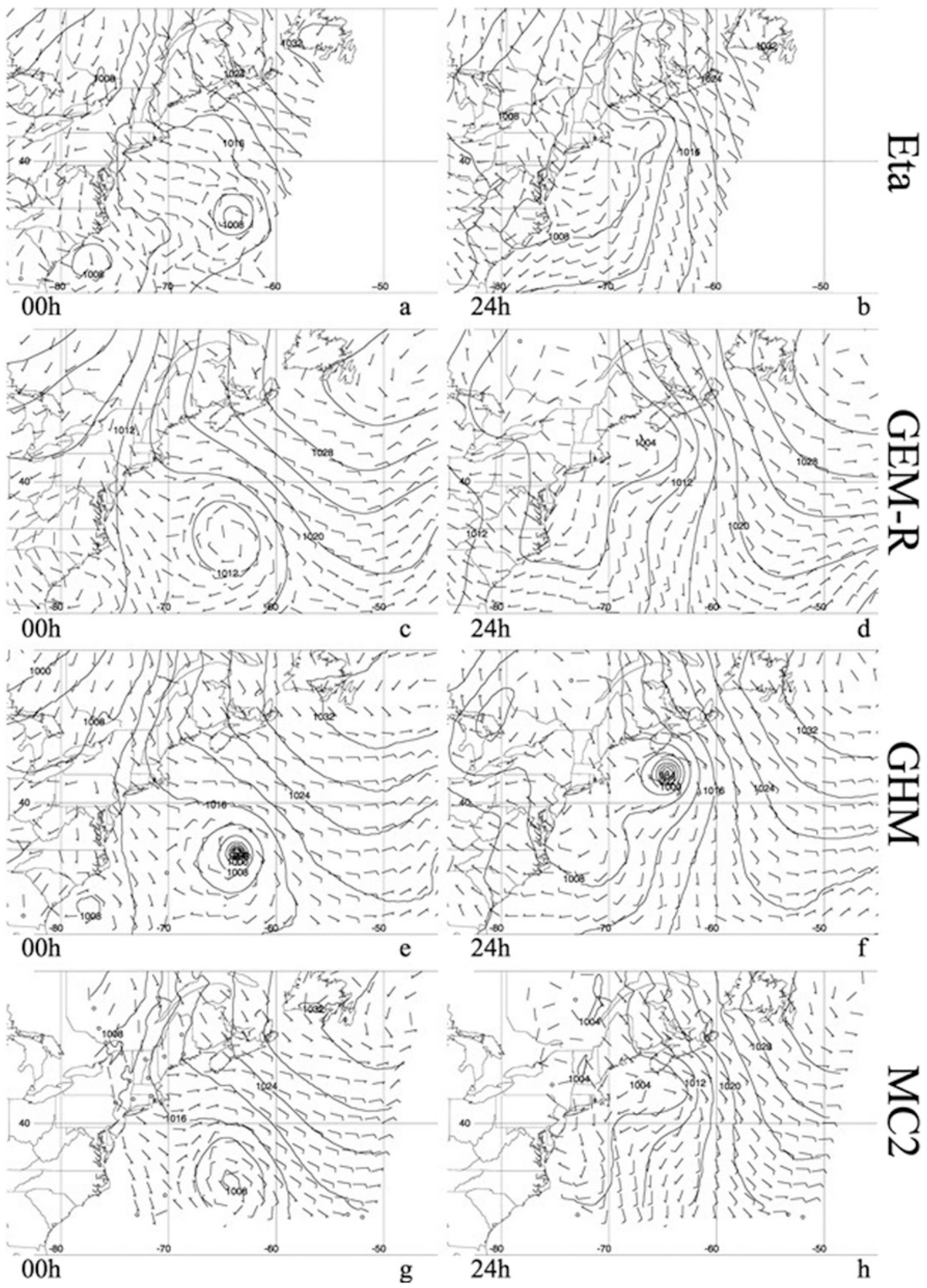

FIG. 15. Selected regional prediction system forecasts for NATL Hurricane Juan initialized at 0000 UTC 28 Sep 2003. Sea level pressure (solid lines; 4-hPa intervals) and winds (barbs; $\mathrm{m} \mathrm{s}^{-1}$ ) are shown for the (left) initial state and (right) 24-h forecasts valid at 0000 UTC 29 Sep 2003. Model fields are indicated for the (a),(b) NCEP ETA Model; (c),(d) regional version of the Environment Canada Global Environmental Multiscale model (GEM-R); (e),(f) GFDL Hurricane Model (GHM); and (g),(h) Mesoscale Compressible Community (MC2) model. Minimum MSLP contours are $984 \mathrm{hPa}$ in (e) and (f). Please see McTaggart-Cowan et al. (2006a) for relevant model details. Figure reproduced from McTaggart-Cowan et al. (2006a, their Fig. 6). 
vortex initialization techniques are unlikely to be generally valid at relatively high latitudes (McTaggartCowan et al. 2006a). Similar conclusions regarding vortex initialization were reached for Juan (2003) by Fogarty et al. (2006) and Michael (2000) by Fogarty et al. (2007). It should be emphasized, however, that the results of these studies are a decade old, and thus the application of more recent data assimilation and numerical modeling systems to this case may produce different conclusions.

In this vein, case studies of NATL TCs Nadine (2012) and Sandy (2012) using modern data assimilation and ensemble forecast systems have highlighted additional causes of medium-range forecast track sensitivity during ET. For Nadine, the forecast track was highly sensitive to the intensity of a midlatitude trough (Munsell et al. 2015) and whether the trough became cut off from the midlatitude flow and engaged in Fujiwhara (1931)-like vortex interaction with Nadine (Pantillon et al. 2016). For Sandy, the results were modeling system dependent, with studies highlighting sensitivity in track and ET timing to slight initial steering-level flow differences in the deep tropics (Munsell and Zhang 2014), downstream midlatitude ridge amplification driven by diabatic processes (Torn et al. 2015), and model configuration (Bassill 2014; Magnusson et al. 2014). As with track, ensemble forecasts of cyclone intensity during ET can be sensitive to both tropical and midlatitude synoptic-scale features in the TC environment (e.g., Doyle et al. 2011). Together, these studies highlight that ET forecast sensitivities vary from one event to the next, particularly as related to tropical versus extratropical influences.

A unifying theory for the joint influences of tropical and extratropical features on TC forecast track sensitivity during ET is provided by the concept of bifurcation points (Scheck et al. 2011). A bifurcation occurs when a small change in one interaction parameter (e.g., midlatitude trough or TC vortex structure) results in a significant change in interaction outcome. The vortex phasing sensitivity highlighted in the idealized simulations of Ritchie and Elsberry (2007) is one practical manifestation of the influence of bifurcation points during ET. When a cyclone approaches such a bifurcation point, small initial track errors will amplify greatly with time (e.g., Emanuel 2005, his Fig. 20.2). Bifurcation points are defined by where the local velocity is zero, such as at the base of a midlatitude trough or apex of a midlatitude ridge (e.g., Riemer and Jones 2014, their Fig. 11). The approach of a TC to such a point can govern whether or not it will recurve and undergo ET as well as its posttransition outcome (Grams et al. 2013; Riemer and Jones 2014; Part II).
Despite advanced understanding of the causes of track forecast sensitivity during ET, it is fair to ask: What can be done to reduce it? Although generally limited to field campaigns, the assimilation of targeted observations into numerical model initial conditions offers promise for improving cyclone representation, reducing track-forecast sensitivity, and reducing track-forecast error prior to and during ET. The assimilation of targeted dropsonde observations collected for WNP TCs Sinlaku and Jangmi during T-PARC in 2008 had a small (Harnisch and Weissmann 2010) to positive (Jung et al. 2012) impact to initial position analyses and subsequent track forecasts compared to experiments only assimilating conventional observations. This improvement resulted not because of targeted observation assimilation during ET, but because of the aggregate positive impact upon the model first-guess field from assimilating targeted observations earlier in the TC life cycle (Weissmann et al. 2011). Targeted lidar-derived water vapor observations improved analysis quality but had a negligible mean impact on forecast quality for the eight T-PARC missions considered by Harnisch et al. (2011). The effects of assimilating Doppler wind lidar observations collected during T-PARC varied, as they had a positive impact at all lead times on TC track and large-scale geopotential height forecasts when collected during recurvature, a small positive impact at $>3$-day lead times when collected during ET, and negligible impact thereafter (Weissmann et al. 2012). Finally, assimilating atmospheric motion vectors improved track forecasts for Sinlaku and Jangmi during T-PARC in 2008 during recurvature by improving midlatitude flow and subtropical anticyclone representation, respectively (Berger et al. 2011; Reynolds et al. 2013).

\section{Future directions}

Despite the substantial advances in ET-related understanding described earlier in this paper, particularly toward addressing the research needs posed by Jones et al. (2003), there remain significant gaps in our understanding of and ability to accurately predict ET. Addressing these gaps will require targeted research across the physical and social sciences, as well as the active engagement of researchers, operational forecasters, hazard communicators, and the public.

Though the CPS has gained widespread acceptance at operational forecast centers in recent years and now serves as a de facto standard for defining ET, it does not resolve a cyclone's inner-core structure and is only as reliable and accurate as its inputs. For example, the $0.5^{\circ}$ latitude-longitude Climate Forecast System Reanalysis (CFSR; Saha et al. 2010) demonstrates discontinuities in 
the mass fields near a TC (e.g., Wood and Ritchie 2014b, their Fig. 6), and the $1.25^{\circ}$ latitude-longitude Japanese 55year Reanalysis (JRA-55; Kobayashi et al. 2015) inconsistently depicts TC size and intensity, particularly at higher latitudes (Harada et al. 2016). Further, a subset of TCs that undergo ET appear to not follow the archetypical CPS pathway of Evans and Hart (2003). Efforts to improve the quality of CPS input data, whether for realtime analysis or retrospective climatology determination, are recommended, as is the continued evaluation of other classifiers for cyclone phase classification.

That being said, given recent improvements to TC observing capabilities and reanalysis product reliability, as well as improved ET understanding, a number of basins would benefit from new, expanded, and/or updated climatologies. In particular, climatologies for the southeast Indian and South Pacific Ocean basins have not been updated in 15-25 years. Further, despite the low climatological TC frequency, potential South Atlantic Ocean ET events identified by several case studies (McTaggart-Cowan et al. 2006b; Evans and Braun 2012; Dias Pinto et al. 2013; Gozzo et al. 2014) motivate further evaluation of ET occurrences in this basin. More generally, a global ET climatology with consistent classification method, input candidate and analysis datasets, and cyclone characteristics has yet to be obtained. While such a study may provide results comparable to previous work, a global climatology may improve understanding of ET variability between basins. Related efforts should seek to diagnose intraseasonal variability from ET climatologies and to better understand the causes of, and resultant impacts from, projected future changes in ET climatologies.

Notable advances in understanding of the ET-related direct impacts of wind, waves, and precipitation have been made in recent years, yet much remains unknown about the physical processes that result in case-to-case variability in these hazards. For example, the causes of left-of-track wind maxima distributions during ET remain uncertain. Further, although recent investigations have evaluated the influence of the air-sea interface on cyclone intensity, these studies have focused on a limited sample of cases during either (and not the transition between) the tropical or extratropical phase. Additional in situ observations are needed to better understand how the atmosphere impacts the ocean, and vice versa, during ET, and thus motivate the development of improved model representations of the relevant physical processes, including wind effects on waves, wave breaking, nonlinear wave-wave interactions, wavebottom friction, and wave-current interactions.

Though it is well understood that decreasing SST, strong vertical wind shear, and/or dry-air infiltration can weaken
TCs, it remains unclear to what extent these processes result in reduced TC intensity during ET, when baroclinic and frontogenetic processes become important to cyclone maintenance and structure. Given the propensity for ET to occur within environments characterized by sharp horizontal kinematic and thermodynamic gradients, and near coastlines, further research across a broad spectrum of ET events is needed to advance understanding of the variability of rapid TC intensity change during ET and the extratropical stage.

Recent and ongoing field campaigns have provided the first comprehensive in situ observational datasets of structural evolution during ET. These data cover a very limited number of cases, however, and thus only a subset of ET variability has been sampled. Other observational platforms are being exploited, particularly microwave retrievals from polar-orbiting satellites and next-generation geostationary satellite imagers, to aid cyclone structural evolution analysis. Continued efforts should be made to best use the data provided by both field campaigns and remote sensing platforms to aid in diagnosing and understanding case-to-case variability in structural evolution during ET. The utility of such data toward the development of improved intensity estimation methods applicable during ET should also be explored.

Despite the heavy reliance by operational forecast centers on model output for ET forecasts, the extent to which recent improvements in numerical models, ensemble methods, and data assimilation techniques have improved ET-related forecast skill is unknown. This is particularly true for the ET-related hazards of wind, waves, and precipitation, as well as the specific forecast elements of cyclone intensity, classification, and thermal structure throughout ET. Research is needed to quantify the practical predictability of these hazards and forecast elements, determine observational and modeling system sensitivities inherent to their prediction, and diagnose case-to-case variability in forecast skill over a large sample of events. It is expected that ensemble methods will be of great use to such investigations, and continued improvements to ensemble data assimilation and modeling systems to support such studies and operational forecasts are advocated. Fundamentally, however, no studies have yet attempted to quantify the intrinsic predictability of ET. Such research may illuminate the forecast system element(s) that, if improved, would provide the most significant and/or cost-effective forecast skill improvements.

In addition to improved understanding of ET and its predictability, engagement with social scientists is needed to best communicate operational ET-related forecasts to end users to promote increased preparedness and response to messages. Indeed, end users 
can perceive ET to be associated with diminished, downgraded, or uncertain threats (Gyakum and Keller 2014; Meyer et al. 2014). Yet, as recently exemplified by NATL TC Sandy (2012), ET is not always associated with reduced impacts, particularly given the areal expansion and increasingly asymmetric distributions of heavy precipitation, high wind speeds, and large waves that often accompany ET. Effective communication therefore requires a focus upon the cyclone's attendant threats rather than its energetics or operational classification (NOAA 2016). It also requires consistent messaging as to the nature of the threat(s): timing and location, the probability of and uncertainty in the expected outcome [such as conveyed in the prototype depicted by Fig. 20 of Gyakum and Keller (2014)], and the actions to take to prepare for and recover from the threat (e.g., NOAA 2013, 2016). How best to accomplish these goals will require further research into how end users receive, perceive, and act upon ET-related forecast information.

The ET research that has been conducted since the publication of Jones et al. (2003) has led to dramatic improvements in our ability to classify ET, advanced understanding of the causes behind and variation in a cyclone's evolving hazards during ET, provided observational evidence of structural evolution to support prior conceptual models, and contributed to better insight into ET-related cyclone track forecast sensitivities. Continued development of observational, modeling, and analysis techniques has the potential to further advance our ability to predict ET and its hazards. The full realization of these potential benefits, however, will require collaborative research between researchers and operational forecasters to effectively translate such advancements into improved forecast skill and engagement with social scientists to effectively translate improved forecast skill into better end-user actions. It is our hope that this review provides a framework to motivate such research as well as other research that is needed to fill the other substantial remaining gaps in ET-related knowledge.

Acknowledgments. We gratefully acknowledge Monthly Weather Review Chief Editor David Schultz for helpful feedback to the preparation process and a comprehensive review of an earlier draft of this review. Feedback from Julia Keller and five anonymous reviewers greatly improved the quality of this review. The authors gratefully acknowledge the support of the National Science Foundation via Grants AGS-1240502 and AGS-1355960 (Bosart); National Oceanic and Atmospheric Administration via Awards NA13NWS4680004 and NA16NWS468005 (Corbosiero) and through its sponsorship of the Hurricane Forecast Improvement Program (Zhang); the Swiss National Science Foundation under Grant PZ00P2_148177/1 (Grams); a Natural Sciences and Engineering Research Council of Canada Discovery Grant (Gyakum); the subproject "Evolution and predictability of storm structure during extratropical transition of tropical cyclones" of the Transregional Collaborative Research Center SFB/TRR 165 "Waves to Weather" program funded by the German Science Foundation (Riemer); and Ouranos (the regional climate consortium of Quebec), the Natural Sciences and Engineering Research Council of Canada, and the Canadian Foundation for Climate and Atmospheric Sciences (Milrad). The National Center for Atmospheric Research is sponsored by the National Science Foundation. The views in this manuscript are those of the authors alone and not of any funding agency or employer. Authors Evans and Wood served as leads for the review; authors Aberson, Archambault, and Milrad served as section leads; and remaining authors served as contributors to the review.

\section{REFERENCES}

Aberson, S. D., 2014: A climatological baseline for assessing the skill of tropical cyclone phase forecasts. Wea. Forecasting, 29 , 122-129, doi:10.1175/WAF-D-12-00130.1.

_ - A. Aksoy, K. J. Sellwood, T. Vukicevic, and X. Zhang, 2015: Assimilation of high-resolution tropical cyclone observations with an ensemble Kalman filter using HEDAS: Evaluation of 2008-11 HWRF forecasts. Mon. Wea. Rev., 143, 511-523, doi:10.1175/MWR-D-14-00138.1.

Abraham, J., J. W. Strapp, C. Fogarty, and M. Wolde, 2004: Extratropical transition of Hurricane Michael: An aircraft investigation. Bull. Amer. Meteor. Soc., 85, 1323-1339, doi:10.1175/BAMS-85-9-1323.

Adams, D. K., and A. C. Comrie, 1997: The North American monsoon. Bull. Amer. Meteor. Soc., 78, 2197-2213, doi:10.1175/1520-0477(1997)078<2197:TNAM>2.0.CO;2.

Allard, R. A., 1984: A climatology of the characteristics of tropical cyclones in the Northeast Pacific during the period 1966-1980. M.S. thesis, Dept. of Geosciences, Texas Tech University, Lubbock, TX, 106 pp.

Archambault, H. M., L. F. Bosart, D. Keyser, and J. M. Cordeira, 2013: A climatological analysis of the extratropical flow response to recurving western North Pacific tropical cyclones. Mon. Wea. Rev., 141, 2325-2346, doi:10.1175/MWR-D-12-00257.1.

Arnott, J. M., J. L. Evans, and F. Chiaromonte, 2004: Characterization of extratropical transition using cluster analysis. Mon. Wea. Rev., 132, 2916-2937, doi:10.1175/MWR2836.1.

Atallah, E. H., and L. F. Bosart, 2003: The extratropical transition and precipitation distribution of Hurricane Floyd (1999). Mon. Wea. Rev., 131, 1063-1081, doi:10.1175/ 1520-0493(2003)131<1063:TETAPD>2.0.CO;2.

,,- and A. R. Aiyyer, 2007: Precipitation distribution associated with landfalling tropical cyclones over the eastern United States. Mon. Wea. Rev., 135, 2185-2206, doi:10.1175/ MWR3382.1. 
Baatsen, M., R. J. Haarsma, A. J. Van Delden, and H. de Vries, 2015: Severe autumn storms in future western Europe with a warmer Atlantic Ocean. Climate Dyn., 45, 949-964, doi:10.1007/s00382-014-2329-8.

Barnes, E. A., L. M. Polvani, and A. H. Sobel, 2013: Model projections of atmospheric steering of Sandy-like superstorms. Proc. Natl. Acad. Sci. USA, 110, 15 211-15215, doi:10.1073/ pnas.1308732110.

Bassill, N., 2014: Accuracy of early GFS and ECMWF Sandy (2012) track forecasts: Evidence for a dependence on cumulus parameterization. Geophys. Res. Lett., 41, 3274-3281, doi:10.1002/2014GL059839.

Bell, M. M., M. T. Montgomery, and K. E. Emanuel, 2012: Air-sea enthalpy and momentum exchange at major hurricane wind speeds observed during CBLAST. J. Atmos. Sci., 69, 31973222, doi:10.1175/JAS-D-11-0276.1.

Berger, H., R. Langland, C. S. Velden, C. A. Reynolds, and P. M. Pauley, 2011: Impact of enhanced satellite-derived atmospheric motion vector observations on numerical tropical cyclone track forecasts in the Western North $\mathrm{Pa}$ cific during TPARC/TCS-08. J. Appl. Meteor. Climatol., 50, 2309-2318, doi:10.1175/JAMC-D-11-019.1.

Beven, J. L., 2012a: RGB airmass imagery: a new tool to diagnose extratropical transition of tropical cyclones. Proc. Fourth Int. Workshop on Extratropical Transition, Sainte-Adele, Quebec, Canada, World Meteorological Organization, 4.1, https:// www.mcgill.ca/meteo/files/meteo/4_1_beven.pdf.

- 2012b: An update on verification of NHC forecasts of extratropical transition. Proc. Fourth Int. Workshop on Extratropical Transition, Sainte-Adele, Quebec, Canada, World Meteorological Organization, 8.1, https://www.mcgill.ca/ meteo/files/meteo/8_1_beven_et_al.pdf.

Black, M. L., J. F. Gamache, F. D. Marks, C. E. Samsury, and H. E. Willoughby, 2002: Eastern Pacific Hurricanes Jimena of 1991 and Olivia of 1994: The effect of vertical shear on structure and intensity. Mon. Wea. Rev., 130, 2291-2312, doi:10.1175/ 1520-0493(2002)130<2291:EPHJOA > 2.0.CO;2.

Blake, E. S., T. B. Kimberlain, R. J. Berg, J. P. Cangialosi, and J. L. Beven III, 2013: Tropical Cyclone Report: Hurricane Sandy (AL182012). Tech. Rep. AL182012, NOAA/National Hurricane Center, 157 pp., http://www.nhc.noaa.gov/data/tcr/ AL182012_Sandy.pdf.

Bosart, L. F., and D. B. Dean, 1991: The Agnes rainstorm of June 1972: Surface feature evolution culminating in inland storm redevelopment. Wea. Forecasting, 6, 515-537, doi:10.1175/ 1520-0434(1991)006<0515:TAROJS >2.0.CO;2.

Bougeault, P., and Coauthors, 2010: The THORPEX Interactive Grand Global Ensemble (TIGGE). Bull. Amer. Meteor. Soc. 91, 1059-1072, doi:10.1175/2010BAMS2853.1.

Bowyer, P. J., and A. W. MacAfee, 2005: The theory of trapped-fetch waves with tropical cyclones-An operational perspective. Wea. Forecasting, 20, 229-244, doi:10.1175/WAF849.1.

Brennan, M. J., C. C. Hennon, and R. D. Knabb, 2009: The operational use of QuikSCAT ocean surface vector winds at the National Hurricane Center. Wea. Forecasting, 24, 621-645, doi:10.1175/2008WAF2222188.1.

Browning, K. A., 1990: Organization of clouds and precipitation in extratropical cyclones. Extratropical Cyclones: The Erik Palmén Memorial Volume, C. W. Newton and E. O. Holopainen, Eds., Amer. Meteor. Soc., 129-153.

_ 2004: The sting at the end of the tail: Damaging winds associated with extratropical cyclones. Quart. J. Roy. Meteor. Soc., 130, 375-399, doi:10.1256/qj.02.143.
Brun, J., and A. P. Barros, 2014: Mapping the role of tropical cyclones on the hydroclimate of the southeast United States: 2002-2011. Int. J. Climatol., 34, 494-517, doi:10.1002/joc.3703.

Bruneau, N., J. Grieser, T. Loridan, E. Bellone, and S. Khare, 2017: The impact of extra-tropical transitioning on storm surge and waves in catastrophe risk modelling: Application to the Japanese coastline. Nat. Hazards, 85, 649-667, doi:10.1007/ s11069-016-2596-2.

Buckingham, C., T. Marchok, I. Ginis, L. Rothstein, and D. Rowe, 2010: Short- and medium-range prediction of tropical and transitioning cyclone tracks within the NCEP Global Ensemble Forecasting System. Wea. Forecasting, 25, 17361754, doi:10.1175/2010WAF2222398.1.

Buizza, R., D. Richardson, and T. N. Palmer, 2001: The new 80-km high-resolution ECMWF EPS. ECMWF Newsletter, No. 90 (Spring), ECMWF, Reading, United Kingdom, 2-9, https://www. ecmwf.int/sites/default/files/elibrary/2001/14634-newsletter-no90spring-2001.pdf.

- , P. L. Houtekamer, G. Pellerin, Z. Toth, Y. Zhu, and M. Wei, 2005: A comparison of the ECMWF, MSC, and NCEP global ensemble prediction systems. Mon. Wea. Rev., 133, 1076-1097, doi:10.1175/MWR2905.1.

Carlson, T. N., 1980: Airflow through midlatitude cyclones and the comma cloud pattern. Mon. Wea. Rev., 108, 1498-1509, doi:10.1175/1520-0493(1980)108<1498:ATMCAT>2.0.CO;2.

Carr, F. H., and L. Bosart, 1978: A diagnostic evaluation of rainfall predictability for Tropical Storm Agnes, June 1972. Mon. Wea. Rev., 106, 363-374, doi:10.1175/ 1520-0493(1978)106<0363:ADEORP > 2.0.CO;2.

Chen, G. H., 2011: A comparison of precipitation distribution of two landfalling tropical cyclones during the extratropical transition. Adv. Atmos. Sci., 28, 1390-1404, doi:10.1007/s00376-011-0148-y.

Chen, S. S., J. A. Knaff, and F. D. Marks Jr., 2006: Effects of vertical wind shear and storm motion on tropical cyclone rainfall asymmetries deduced from TRMM. Mon. Wea. Rev., 134, 3190-3208, doi:10.1175/MWR3245.1.

Corbosiero, K. L., and J. Molinari, 2002: The effects of vertical wind shear on the distribution of convection in tropical cyclones. Mon. Wea. Rev., 130, 2110-2123, doi:10.1175/ 1520-0493(2002)130<2110:TEOVWS $>2.0 . \mathrm{CO} ; 2$.

— M. J. Dickinson, and L. F. Bosart, 2009: The contribution of eastern North Pacific tropical cyclones to the rainfall climatology of the southwest United States. Mon. Wea. Rev., 137, 2415-2435, doi:10.1175/2009MWR2768.1.

Coumou, D., V. Pethokhov, S. Rahmstorf, S. Petri, and H. J. Schellnhuber, 2014: Quasi-resonant circulation regimes and hemispheric synchronization of extreme weather in boreal summer. Proc. Natl. Acad. Sci. USA, 111, 12331-12336, doi:10.1073/pnas.1412797111.

Davis, C. A., S. C. Jones, and M. Riemer, 2008: Hurricane vortex dynamics during Atlantic extratropical transition. J. Atmos. Sci., 65, 714-736, doi:10.1175/2007JAS2488.1.

W. Wang, J. Dudhia, and R. Torn, 2010: Does increased horizontal resolution improve hurricane wind forecasts? Wea. Forecasting, 25, 1826-1841, doi:10.1175/2010WAF2222423.1.

DeHart, J. C., R. A. Houze Jr., and R. F. Rogers, 2014: Quadrant distribution of tropical cyclone inner-core kinematics in relation to environmental shear. J. Atmos. Sci., 71, 2713-2732, doi:10.1175/JAS-D-13-0298.1.

Demirci, O., J. S. Tyo, and E. A. Ritchie, 2007: Spatial and spatiotemporal projection pursuit techniques to predict the extratropical transition of tropical cyclones. IEEE Trans. Geosci. Remote Sens., 45, 418-424, doi:10.1109/TGRS.2006.882251. 
Dias Pinto, J. R., M. S. Reboita, and R. P. da Rocha, 2013: Synoptic and dynamical analysis of subtropical cyclone Anita (2010) and its potential for tropical transition over the South Atlantic Ocean. J. Geophys. Res. Atmos., 118, 10 870-10 883, doi:10.1002/ jgrd.50830.

Dickinson, M., L. Bosart, K. Corbosiero, S. Hopsch, K. Lombardo, M. Novak, B. Smith, and A. Wasula, 2004: The extratropical transitions of eastern Pacific Hurricane Lester (1992) and Atlantic Hurricane Andrew (1992): A comparison. 26th Conf. on Hurricanes and Tropical Meteorology, Miami, FL, Amer. Meteor. Soc., 17D.2, https://ams.confex.com/ams/26HURR/ techprogram/paper_75685.htm.

Doyle, J. D., C. A. Reynolds, and C. Amerault, 2011: Diagnosing tropical cyclone sensitivity. Comput. Sci. Eng., 13, 31-39, doi:10.1109/MCSE.2010.146.

Dudhia, J., 1993: A nonhydrostatic version of the Penn StateNCAR mesoscale model: Validation tests and simulation of an Atlantic cyclone and cold front. Mon. Wea. Rev., 121, 1493-1513, doi:10.1175/1520-0493(1993)121<1493:ANVOTP>2.0.CO;2.

Dvorak, V., 1984: Tropical cyclone intensity analysis using satellite data. NOAA Tech. Rep. NESDIS11, 47 pp. [Available from NOAA/NESDIS, 5200 Auth Rd., Washington, DC 20233.]

Elsberry, R. L., and P. A. Harr, 2008: Tropical Cyclone Structure (TCS08) field experiment science basis, observational platforms, and strategy. Asia-Pac. J. Atmos. Sci., 44, 209-231.

Emanuel, K., 2005: Divine Wind: The History and Science of Hurricanes. Oxford University Press, 296 pp.

Evans, C., and R. E. Hart, 2008: Analysis of the wind field evolution associated with the extratropical transition of Bonnie (1998). Mon. Wea. Rev., 136, 2047-2065, doi:10.1175/ 2007MWR2051.1.

Evans, J. L., and R. E. Hart, 2003: Objective indicators of the life cycle evolution of extratropical transition for Atlantic tropical cyclones. Mon. Wea. Rev., 131, 909-925, doi:10.1175/ 1520-0493(2003)131<0909:OIOTLC >2.0.CO;2.

— transition intensification of Hurricane Irene (1999). Mon. Wea. Rev., 132, 1355-1368, doi:10.1175/1520-0493(2004)132<1355: FATPIO $>2.0 . \mathrm{CO} ; 2$.

— the South Atlantic. J. Climate, 25, 7328-7340, doi:10.1175/ JCLI-D-11-00212.1.

J. M. Arnott, and F. Chiaromonte, 2006: Evaluation of operational model cyclone structure forecasts during extratropical transition. Mon. Wea. Rev., 134, 3054-3072, doi:10.1175/MWR3236.1.

Farfàn, L. M., 2004: Regional observations during the landfall of Tropical Cyclone Juliette (2001) in Baja California, Mexico. Mon. Wea. Rev., 132, 1575-1589, doi:10.1175/ 1520-0493(2004)132<1575:RODTLO > 2.0.CO;2.

Foerster, A. M., M. M. Bell, P. A. Harr, and S. C. Jones, 2014: Observations of the eyewall structure of Typhoon Sinlaku (2008) during the transformation stage of extratropical transition. Mon. Wea. Rev., 142, 3372-3392, doi:10.1175/MWR-D-13-00313.1.

Fogarty, C., 2006: The extratropical transition of Tropical Storm Ophelia (2005): Summary of forecasts and meteorological observations. 27th Conf. on Hurricanes and Tropical Meteorology, Monterey, CA, Amer. Meteor. Soc., P6.1, https://ams. confex.com/ams/27Hurricanes/techprogram/paper_107911.htm.

_ 2010: Forecasting extratropical transition. Proc. Seventh Int. Workshop on Tropical Cyclones, St. Gilles Les Bains, La Reunion, France, World Meteorological Organization, 2.5, http://www.wmo.int/pages/prog/arep/wwrp/tmr/otherfileformats/ documents/2_5.pdf.
_ and E. Blake, 2013: The double life of Hurricane Sandy and a climatological perspective of these post-tropical giants [in "State of the Climate in 2012"]. Bull. Amer. Meteor. Soc., 94, S109-S110.

_ R. J. Greatbatch, and H. Ritchie, 2006: The role of anomalously warm sea surface temperatures on the intensity of Hurricane Juan (2003) during its approach to Nova Scotia Mon. Wea. Rev., 134, 1484-1504, doi:10.1175/MWR3140.1.

$\longrightarrow, \ldots$, and — 2007: The use of a vortex insertion technique to simulate the extratropical transition of Hurricane Michael (2000). Wea. Forecasting, 22, 480-500, doi:10.1175/ WAF1014.1.

Foley, G. R., and B. N. Hanstrum, 1994: The capture of tropical cyclones by cold fronts off the west coast of Australia. Wea. Forecasting, 9, 577-592, doi:10.1175/1520-0434(1994)009<0577: ТСОТСВ $>2.0 . \mathrm{CO} ; 2$.

Francis, J. A., and S. J. Vavrus, 2015: Evidence for a wavier jet stream in response to rapid Arctic warming. Environ. Res. Lett., 10, 014005, doi:10.1088/1748-9326/10/1/014005.

Frank, W. M., and E. A. Ritchie, 2001: Effects of vertical wind shear on the intensity and structure of numerically simulated hurricanes. Mon. Wea. Rev., 129, 2249-2269, doi:10.1175/ 1520-0493(2001)129<2249:EOVWSO > 2.0.CO;2.

Fujibe, F., and N. Kitabatake, 2007: Classification of surface wind fields of tropical cyclones at landfall on the Japan main islands. J. Meteor. Soc. Japan, 85, 747-765, doi:10.2151/jmsj.85.747.

,,-- K. Bessho, and S. Hoshino, 2006: Comparison of surface wind fields in Typhoon 0418 (Songda) and Typhoon 9119 (Mireille) in western Japan. Pap. Meteor. Geophys., 57, 1-9, doi:10.2467/mripapers.57.1.

Fujiwhara, S., 1931: Short note on the behaviour of two vortices. Proc. Phys. Math Soc. Japan, Ser. 3, 13, 106-110.

Galarneau, T. J., Jr., L. F. Bosart, and R. S. Schumacher, 2010: Predecessor rain events ahead of tropical cyclones. Mon. Wea. Rev., 138, 3272-3297, doi:10.1175/2010MWR3243.1.

— C. A. Davis, and M. A. Shapiro, 2013: Intensification of Hurricane Sandy (2012) through extratropical warm core seclusion. Mon. Wea. Rev., 141, 4296-4321, doi:10.1175/ MWR-D-13-00181.1.

Garde, L. A., A. B. Pezza, I. Simmonds, and N. E. Davidson, 2010: A methodology of tracking transitioning Cyclones. IOP Conf. Ser.: Earth Environ. Sci., 11, 012007, doi:10.1088/1755-1315/11/ $1 / 012007$.

Gozzo, L. F., R. P. da Rocha, M. S. Reboita, and S. Sugahara, 2014: Subtropical cyclones over the southwestern South Atlantic: Climatological aspects and case study. J. Climate, 27, 85438562, doi:10.1175/JCLI-D-14-00149.1.

Grams, C. M., and S. R. Blumer, 2015: European high-impact weather caused by the downstream response to the extratropical transition of North Atlantic Hurricane Katia (2011). Geophys. Res. Lett., 42, 8738-8748, doi:10.1002/2015GL066253.

_ S. C. Jones, and C. A. Davis, 2013: The impact of Typhoon Jangmi (2008) on the midlatitude flow. Part II: Downstream evolution. Quart. J. Roy. Meteor. Soc., 139, 2165-2180, doi:10.1002/qj.2119.

Griffin, K. S., and L. F. Bosart, 2014: The extratropical transition of Tropical Cyclone Edisoana (1990). Mon. Wea. Rev., 142, 2772-2793, doi:10.1175/MWR-D-13-00282.1.

Gyakum, J., and J. Keller, 2014: Advances in understanding ET. Proc. Eighth Int. Workshop on Tropical Cyclones, Jeju, South Korea, World Meteorological Organization, 4.2, https:// www.wmo.int/pages/prog/arep/wwrp/new/documents/Topic4.2 AdvancesinUnderstaidningET.pdf. 
Haarsma, R. J., W. Hazeleger, C. Severijns, H. de Vries, A. Sterl, R. Bintanja, G. J. van Oldenborgh, and H. W. van den Brink, 2013: More hurricanes to hit western Europe due to global warming. Geophys. Res. Lett., 40, 1783-1788, doi:10.1002/ grl.50360.

Hanley, D., J. Molinari, and D. Keyser, 2001: A composite study of the interactions between tropical cyclones and uppertropospheric troughs. Mon. Wea. Rev., 129, 2570-2584, doi:10.1175/1520-0493(2001)129<2570:ACSOTI>2.0.CO;2.

Harada, Y., and Coauthors, 2016: The JRA-55 reanalysis: Representation of atmospheric circulation and climate variability. J. Meteor. Soc. Japan, 94, 269-302, doi:10.2151/jmsj.2016-015.

Harnisch, F., and M. Weissmann, 2010: Sensitivity of typhoon forecasts to different subsets of targeted dropsonde observations. Mon. Wea. Rev., 138, 2664-2680, doi:10.1175/ 2010MWR3309.1.

— _ C C. Cardinali, and M. Wirth, 2011: Experimental assimilation of DIAL water vapour observations in the ECMWF global model. Quart. J. Roy. Meteor. Soc., 137, 1532-1546, doi:10.1002/qj.851.

Harr, P. A., and R. L. Elsberry, 2000: Extratropical transition of tropical cyclones over the western North Pacific. Part I: Evolution of structural characteristics during the transition process. Mon. Wea. Rev., 128, 2613-2633, doi:10.1175/ 1520-0493(2000)128<2613:ETOTCO > 2.0.CO;2.

— D. Anwender, and S. C. Jones, 2008: Predictability associated with the downstream impacts of the extratropical transition of tropical cyclones: Methodology and a case study of Typhoon Nabi (2005). Mon. Wea. Rev., 136, 3205-3225, doi:10.1175/ 2008MWR2248.1.

Hart, R. E., 2003: A cyclone phase space derived from thermal wind and thermal asymmetry. Mon. Wea. Rev., 131, 585-616, doi:10.1175/1520-0493(2003)131<0585:ACPSDF>2.0.CO;2.

—, and J. L. Evans, 2001: A climatology of the extratropical transition of Atlantic tropical cyclones. J. Climate, 14, 546-564 doi:10.1175/1520-0442(2001)014<0546:ACOTET>2.0.CO;2.

,-- , and C. Evans, 2006: Synoptic composites of the extratropical transition life cycle of North Atlantic tropical cyclones: Factors determining posttransition evolution. Mon. Wea. Rev., 134, 553-578, doi:10.1175/MWR3082.1.

Hill, K. A., and G. M. Lackmann, 2009: Influence of environmental humidity on tropical cyclone size. Mon. Wea. Rev., 137, 3294 3315, doi:10.1175/2009MWR2679.1.

Hodges, K., A. Cobb, and P. L. Vidale, 2017: How well are tropical cyclones represented in reanalysis datasets? J. Climate, $\mathbf{3 0}$ 5243-5264, doi:10.1175/JCLI-D-16-0557.1.

Ito, R., T. Takemi, and O. Arakawa, 2016: A possible reduction in the severity of typhoon wind in the northern part of Japan under global warming: A case study. Sci. Online Lett. Atmos., 12, 100-105.

Jones, S. C., and Coauthors, 2003: The extratropical transition of tropical cyclones: Forecast challenges, current understanding, and future directions. Wea. Forecasting, 18, 1052-1092, doi:10.1175/1520-0434(2003)018<1052:TETOTC $>2.0 . C O ; 2$.

Jung, B.-J., H.-M. Kim, F. Zhang, and C.-C. Wu, 2012: Effect of targeted dropsonde observations and best track data on the track forecasts of Typhoon Sinlaku (2008) using an ensemble Kalman filter. Tellus, 64A, 14984, doi:10.3402/ tellusa.v64i0.14984.

Katsumata, M., S. Mori, B. Geng, and J. Inoue, 2016: Internal structure of ex-Typhoon Phanfone (2014) under an extratropical transition as observed by the research vessel Mirai. Geophys. Res. Lett., 43, 9333-9341, doi:10.1002/2016GL070384.
Keighton, S., D. K. Miller, D. Hotz, P. D. Moore, L. B. Perry, L. G. Lee, and D. T. Martin, 2016: Northwest flow snow aspects of Hurricane Sandy. Wea. Forecasting, 31, 173-195, doi:10.1175/ WAF-D-15-0069.1.

Keller, J. H., S. C. Jones, J. L. Evans, and P. A. Harr, 2011: Characteristics of the TIGGE multimodel ensemble prediction system in representing forecast variability associated with extratropical transition. Geophys. Res. Lett., 38, L12802, doi:10.1029/2011GL047275.

Kitabatake, N., 2008: Extratropical transition of tropical cyclones in the western North Pacific: Their frontal evolution. Mon. Wea. Rev., 136, 2066-2090, doi:10.1175/2007MWR1958.1. 2011: Climatology of extratropical transition of tropical cyclones in the western North Pacific defined by using cyclone phase space. J. Meteor. Soc. Japan, 89, 309-325, doi:10.2151/ jmsj.2011-402.

- and F. Fujibe, 2009: Relationship between surface wind fields and three-dimensional structures of tropical cyclones landfalling in the main islands of Japan. J. Meteor. Soc. Japan, 87, 959-977, doi:10.2151/jmsj.87.959.

Klein, P. M., P. A. Harr, and R. L. Elsberry, 2000: Extratropical transition of western North Pacific tropical cyclones: An overview and conceptual model of the transformation stage. Wea. Forecasting, 15, 373-395, doi:10.1175/ 1520-0434(2000)015<0373:ETOWNP > 2.0.CO;2

- — , and - 2002: Extratropical transition of western North Pacific tropical cyclones: Midlatitude contributions to intensification. Mon. Wea. Rev., 130, 2240-2259, doi:10.1175/ 1520-0493(2002)130<2240:ETOWNP>2.0.CO;2.

Knaff, J. A., D. P. Brown, J. Courtney, G. M. Gallina, and J. L. Beven II, 2010: An evaluation of Dvorak technique-based tropical cyclone intensity estimates. Wea. Forecasting, 25, 1362-1379, doi:10.1175/2010WAF2222375.1.

Kobayashi, S., and Coauthors, 2015: The JRA-55 reanalysis: General specifications and basic characteristics. J. Meteor. Soc. Japan, 93, 5-48, doi:10.2151/jmsj.2015-001.

Kofron, D. E., E. A. Ritchie, and J. S. Tyo, 2010a: Determination of a consistent time for the extratropical transition of tropical cyclones. Part I: Examination of existing methods for finding "ET time." Mon. Wea. Rev., 138, 4328-4343, doi:10.1175/ 2010MWR3180.1.

,$- \longrightarrow$, and $-2010 \mathrm{~b}$ : Determination of a consistent time for the extratropical transition of tropical cyclones Part II: Potential vorticity metrics. Mon. Wea. Rev., 138, 4344-4361, doi:10.1175/2010MWR3181.1.

Kowaleski, A. M., and J. L. Evans, 2016: Regression mixture model clustering of multimodel ensemble forecasts of Hurricane Sandy: Partition characteristics. Mon. Wea. Rev., 144, 38253846, doi:10.1175/MWR-D-16-0099.1.

Kozar, M. E., and V. Misra, 2014: Statistical prediction of integrated kinetic energy in North Atlantic tropical cyclones. Mon. Wea. Rev., 142, 4646-4657, doi:10.1175/MWR-D-14-00117.1.

Landsea, C. W., and J. L. Franklin, 2013: Atlantic hurricane database uncertainty and presentation of a new database format. Mon. Wea. Rev., 141, 3576-3592, doi:10.1175/MWR-D-12-00254.1.

Lentink, H. S., 2017: Mechanisms determining structural changes during the extratropical transition of Typhoon Sinlaku (2008): A modelling study. Ph.D. dissertation, Karlsruhe Institute of Technology, Karlsruhe, Germany, 211 pp.

Li, Q., and Q. Wang, 2013: Re-examination of the potential vorticity metrics for determining extratropical transition onset and completion times using high-resolution data. Acta Meteor. Sin., 27, 502-508, doi:10.1007/s13351-013-0404-4. 
Lin, Y.-L., S. Chiao, T.-A. Wang, M. L. Kaplan, and R. P. Weglarz, 2001: Some common ingredients for heavy orographic rainfall. Wea. Forecasting, 16, 633-660, doi:10.1175/ 1520-0434(2001)016<0633:SCIFHO > 2.0.CO;2.

Liu, M., and J. A. Smith, 2016: Extreme rainfall from landfalling tropical cyclones in the eastern United States: Hurricane Irene (2011). J. Hydrometeor., 17, 2883-2904, doi:10.1175/ JHM-D-16-0072.1.

—, G. A. Vecchi, J. A. Smith, and H. Murakami, 2017: The presentday simulation and twenty-first-century projection of the climatology of extratropical transition in the North Atlantic. J. Climate, 30, 2739-2756, doi:10.1175/JCLI-D-16-0352.1.

Lonfat, M., F. D. Marks Jr., and S. S. Chen, 2004: Precipitation distribution in tropical cyclones using the Tropical Rainfall Measuring Mission (TRMM) Microwave Imager: A global perspective. Mon. Wea. Rev., 132, 1645-1660, doi:10.1175/ 1520-0493(2004)132<1645:PDITCU>2.0.CO;2.

Loridan, T., E. Scherer, M. Dixon, E. Bellone, and S. Khare, 2014: Cyclone wind field asymmetries during extratropical transition in the western North Pacific. J. Appl. Meteor. Climatol., 53, 421428, doi:10.1175/JAMC-D-13-0257.1.

— S. Khare, E. Scherer, M. Dixon, and E. Bellone, 2015: Parametric modeling of transitioning cyclone wind fields for risk assessment studies in the western North Pacific. J. Appl. Meteor. Climatol., 54, 624-642, doi:10.1175/JAMC-D-14-0095.1.

Ma, Z., J. Fei, X. Cheng, Y. Wang, and X. Huang, 2015: Contributions of surface sensible heat fluxes to tropical cyclone. Part II: The sea spray processes. J. Atmos. Sci., 72, 4218-4236, doi:10.1175/JAS-D-15-0058.1.

MacAfee, A. W., and P. J. Bowyer, 2005: The modeling of trapped-fetch waves with tropical cyclones-A desktop operational model. Wea. Forecasting, 20, 245-263, doi:10.1175/ WAF850.1.

—_, and G. M. Pearson, 2006: Development and testing of tropical cyclone parametric wind models tailored for midlatitude application-Preliminary results. J. Appl. Meteor. Climatol., 45, 1244-1260, doi:10.1175/JAM2407.1.

Magnusson, L., J. Bidlot, S. T. Lang, A. Thorpe, N. Wedi, and M. Yamaguchi, 2014: Evaluation of medium-range forecasts for Hurricane Sandy. Mon. Wea. Rev., 142, 1962-1981, doi:10.1175/MWR-D-13-00228.1.

Mahoney, K., and Coauthors, 2016: Understanding the role of atmospheric rivers in heavy precipitation in the southeast United States. Mon. Wea. Rev., 144, 1617-1632, doi:10.1175/ MWR-D-15-0279.1.

Manion, A., C. Evans, T. L. Olander, C. S. Velden, and L. D. Grasso, 2015: An evaluation of advanced Dvorak techniquederived tropical cyclone intensity estimates during extratropical transition using synthetic satellite imagery. Wea. Forecasting, 30, 984-1009, doi:10.1175/WAF-D-15-0019.1.

Mantua, N. J., S. R. Hare, Y. Zhang, J. M. Wallace, and R. C. Francis, 1997: A Pacific interdecadal climate oscillation with impacts on salmon production. Bull. Amer. Meteor. Soc., 78, 1069-1079, doi:10.1175/1520-0477(1997)078<1069: APICOW $>2.0 . \mathrm{CO} ; 2$.

Marks, F. D., Jr., R. A. Houze Jr., and J. F. Gamache, 1992: Dualaircraft investigation of the inner core of Hurricane Norbert. Part I: Kinematic structure. J. Atmos. Sci., 49, 919-942, doi:10.1175/1520-0469(1992)049<0919:DAIOTI>2.0.CO;2.

Mashiko, W., 2008: Formation mechanism of a low-level jet during the passage of Typhoon Ma-on (2004) over the southern Kanto district. J. Meteor. Soc. Japan, 86, 183-202, doi:10.2151/ jmsj.86.183.
Matano, J., 1958: On the synoptic structure of Hurricane Hazel, 1954, over the eastern United States. J. Meteor. Soc. Japan, 36, 23-31, doi:10.2151/jmsj1923.36.1_23.

Matyas, C. J., 2010a: Locating convection in landfalling tropical cyclones: A GIS-based analysis of radar reflectivities and comparison to lightning-based observations. Phys. Geogr., 31, 385-406, doi:10.2747/0272-3646.31.5.385.

- 2010b: Associations between the size of hurricane rain fields at landfall and their surrounding environments. Meteor. Atmos. Phys., 106, 135-148, doi:10.1007/s00703-009-0056-1.

- 2010c: A geospatial analysis of convective rainfall regions within tropical cyclones after landfall. Int. J. Appl. Geospatial Res., 1, 71-91, doi:10.4018/jagr.2010020905.

_ 2013: Processes influencing rain-field growth and decay after tropical cyclone landfall in the United States. J. Appl. Meteor. Climatol., 52, 1085-1096, doi:10.1175/JAMC-D-12-0153.1.

McTaggart-Cowan, R., L. F. Bosart, J. R. Gyakum, and E. H. Atallah, 2006a: Hurricane Juan (2003). Part II: Forecasting and numerical simulation. Mon. Wea. Rev., 134, 1748-1771, doi:10.1175/MWR3143.1.

,,-- C. A. Davis, E. H. Atallah, J. R. Gyakum, and K. A. Emanuel, 2006b: Analysis of Hurricane Catarina (2004). Mon. Wea. Rev., 134, 3029-3053, doi:10.1175/MWR3330.1.

,$- \ldots$, J. R. Gyakum, and E. H. Atallah, 2007: Hurricane Katrina (2005). Part I: Complex life cycle of an intense tropical cyclone. Mon. Wea. Rev., 135, 3905-3926, doi:10.1175/2007MWR1875.1.

Merrill, R. T., 1993: Tropical cyclone structure. Global Guide to Tropical Cyclone Forecasting, WMO/TD-560, Rep. TCP-31, World Meteorological Organization, Geneva, Switzerland, 2.1-2.60.

Meyer, R. J., J. Baker, K. Broad, J. Czajkowski, and B. Orlove, 2014: The dynamics of hurricane risk perception: Real-time evidence from the 2012 Atlantic hurricane season. Bull. Amer. Meteor. Soc., 95, 1389-1404, doi:10.1175/BAMS-D-12-00218.1.

Miller, D. W., and M. A. Lander, 1997: Intensity estimation of tropical cyclones during extratropical transition. JTWC Rep. JTWC/SATOPS/TN-97/002, 9 pp.

Milrad, S. M., E. H. Atallah, and J. R. Gyakum, 2009: Dynamical and precipitation structures of poleward-moving tropical cyclones in eastern Canada, 1979-2005. Mon. Wea. Rev., 137, 836-851, doi:10.1175/2008MWR2578.1.

,$- \ldots$, and $\_, 2013$ : Precipitation modulation by the Saint Lawrence River Valley in association with transitioning tropical cyclones. Wea. Forecasting, 28, 331-352, doi:10.1175/ WAF-D-12-00071.1.

Mokhov, I. I., E. M. Dobryshman, and M. E. Makarova, 2014: Transformation of tropical cyclones into extratropical: The tendencies of 1970-2012. Dokl. Earth Sci., 454, 59-63, doi:10.1134/S1028334X14010127.

Molteni, F., R. Buizza, T. N. Palmer, and T. Petroliagis, 1996: The ECMWF Ensemble Prediction System: Methodology and validation. Quart. J. Roy. Meteor. Soc., 122, 73-119, doi:10.1002/qj.49712252905.

Munsell, E. B., and F. Zhang, 2014: Prediction and uncertainty of Hurricane Sandy (2012) explored through a real-time cloudpermitting ensemble analysis and forecast system assimilating airborne Doppler observations. J. Adv. Model. Earth Syst., 6, 38-58, doi:10.1002/2013MS000297.

_ J. A. Sippel, S. A. Braun, Y. Weng, and F. Zhang, 2015: Dynamics and predictability of Hurricane Nadine (2012) evaluated through convection-permitting ensemble analysis and forecasts. Mon. Wea. Rev., 143, 4514-4532, doi:10.1175/ MWR-D-14-00358.1. 
Ng, B., K. Walsh, and S. Lavender, 2015: The contribution of tropical cyclones to rainfall in northwest Australia. Int. J. Climatol., 35, 2689-2697, doi:10.1002/joc.4148.

NOAA, 2013: Hurricane/Post-Tropical Cyclone Sandy, October 22-29, 2012. NWS service assessment, 66 pp., http://www. weather.gov/media/publications/assessments/Sandy13.pdf.

- 2016: Risk communication and behavior: Best practices and research findings. NOAA Social Science Committee, 66 pp. http://www.performance.noaa.gov/wp-content/uploads/RiskCommunication-and-Behavior-Best-Practices-and-ResearchFindings-July-2016.pdf.

Olander, T. L., and C. S. Velden, 2007: The advanced Dvorak technique: Continued development of an objective scheme to estimate tropical cyclone intensity using geostationary infrared satellite imagery. Wea. Forecasting, 22, 287-298, doi:10.1175/WAF975.1.

Palmén, E., 1958: Vertical circulation and release of kinetic energy during the development of hurricane Hazel into an extratropical storm. Tellus, 10,1-23, doi:10.3402/tellusa.v10i1.9222.

Pantillon, F., J.-P. Chaboureau, C. Lac, and P. Mascart, 2013: On the role of a Rossby wave train during the extratropical transition of Hurricane Helene (2006). Quart. J. Roy. Meteor. Soc., 139, 370-386, doi:10.1002/qj.1974.

- - — , and E. Richard, 2016: Vortex-vortex interaction between Hurricane Nadine (2012) and an Atlantic cut-off dropping the predictability over the Mediterranean. Quart. J. Roy. Meteor. Soc., 142, 419-432, doi:10.1002/qj.2635.

Perrie, W., E. L Andreas, W. Zhang, W. Li, J. Gyakum, and R. McTaggart-Cowan, 2005: Sea spray impacts on intensifying midlatitude cyclones. J. Atmos. Sci., 62, 1867-1883, doi:10.1175/JAS3436.1.

Petterssen, S., 1956: Weather Analysis and Forecasting. 2nd ed. Vol. I, McGraw-Hill, 428 pp.

Powell, M. D., 1982: The transition of the Hurricane Frederic boundary-layer wind fields from the open Gulf of Mexico to landfall. Mon. Wea. Rev., 110, 1912-1932, doi:10.1175/ 1520-0493(1982)110<1912:TTOTHF $>2.0$. CO;2.

Qian, W.-H., J. Huang, and J. Du, 2016: Examination of Hurricane Sandy's (2012) structure and intensity evolution from full-field and anomaly-field analyses. Tellus, 68A, 29029, doi:10.3402/ tellusa.v68.29029.

Quinting, J. F., and S. C. Jones, 2016: On the impact of tropical cyclones on Rossby wave packets: A climatological perspective. Mon. Wea. Rev., 144, 2021-2048, doi:10.1175/MWR-D-14-00298.1.

_ , M. M. Bell, P. A. Harr, and S. C. Jones, 2014: Structural characteristics of T-PARC Typhoon Sinlaku during its extratropical transition. Mon. Wea. Rev., 142, 1945-1961, doi:10.1175/MWR-D-13-00306.1.

Reasor, P. D., M. D. Eastin, and J. F. Gamache, 2009: Rapidly intensifying Hurricane Guillermo (1997). Part I: Lowwavenumber structure and evolution. Mon. Wea. Rev., 137, 603-631, doi:10.1175/2008MWR2487.1.

Reynolds, C. A., R. Langland, P. M. Pauley, and C. Velden, 2013: Tropical cyclone data impact studies: Influences of model bias and synthetic observations. Mon. Wea. Rev., 141, 4373-4394, doi:10.1175/MWR-D-12-00300.1.

Riemer, M., and S. C. Jones, 2014: Interaction of a tropical cyclone with a high-amplitude, mid-latitude wave pattern: Waviness analysis, trough deformation and track bifurcation. Quart. J. Roy. Meteor. Soc., 140, 1362-1376, doi:10.1002/qj.2221.

- P. Hofheinz, and S. C. Jones, 2006: Structural changes of low level wind field of tropical cyclones in idealised extratropical transition scenarios. 27th Conf. on Hurricanes and
Tropical Meteorology, Monterey, CA, Amer. Meteor. Soc., P6.8, https://ams.confex.com/ams/27Hurricanes/techprogram/ paper_108714.htm.

Ritchie, E. A., and R. L. Elsberry, 2001: Simulations of the transformation stage of the extratropical transition of tropical cyclones. Mon. Wea. Rev., 129, 1462-1480, doi:10.1175/ 1520-0493(2001)129<1462:SOTTSO > 2.0.CO;2.

- and 2003: Simulations of the extratropical transition of tropical cyclones: Contributions by the midlatitude upper-level trough to reintensification. Mon. Wea. Rev., 131, 2112-2128, doi:10.1175/1520-0493(2003)131<2112: SOTETO $>2.0 . \mathrm{CO} ; 2$.

, and - 2007: Simulations of the extratropical transition of tropical cyclones: Phasing between the upper-level trough and tropical cyclone. Mon. Wea. Rev., 135, 862-876, doi:10.1175/ MWR3303.1.

- K. M. Wood, D. S. Gutzler, and S. White, 2011: The influence of eastern Pacific tropical cyclone remnants on the southwestern United States. Mon. Wea. Rev., 139, 192-210, doi:10.1175/2010MWR3389.1.

Rogers, R. F., and Coauthors, 2006: The Intensity Forecasting Experiment: A NOAA multiyear field program for improving tropical cyclone intensity forecasts. Bull. Amer. Meteor. Soc., 87, 1523-1537, doi:10.1175/BAMS-87-11-1523.

, and Coauthors, 2013: NOAA's Hurricane Intensity Forecasting Experiment: A progress report. Bull. Amer. Meteor. Soc., 94, 859-882, doi:10.1175/BAMS-D-12-00089.1.

Saha, S., and Coauthors, 2010: The NCEP Climate Forecast System Reanalysis. Bull. Amer. Meteor. Soc., 91, 1015-1057, doi:10.1175/2010BAMS3001.1.

Satake, Y., M. Inatsu, M. Mori, and A. Hasegawa, 2013: Tropical cyclone tracking using a neighbor enclosed area tracking algorithm. Mon. Wea. Rev., 141, 3539-3555, doi:10.1175/ MWR-D-12-00092.1.

Scheck, L., S. C. Jones, and M. Juckes, 2011: The resonant interaction of a tropical cyclone and a tropopause front in a barotropic model. Part II: Frontal waves. J. Atmos. Sci., 68, 405-419, doi:10.1175/2010JAS3482.1.

Semmler, T., S. Varghese, R. McGrath, P. Nolan, S. Wang, P. Lynch, and C. O'Dowd, 2008: Regional model simulation of North Atlantic cyclones: Present climate and idealized response to increased sea surface temperature. J. Geophys. Res., 113, D02107, doi:10.1029/2006JD008213.

Shapiro, M. A., and D. Keyser, 1990: Fronts, jet streams, and the tropopause. Extratropical Cyclones: The Erik Palmen Memorial Volume, C. W. Newton and E. O. Holopainen, Eds., Amer. Meteor. Soc., 167-191.

Shin, J. H., and D.-L. Zhang, 2017: The impact of moist frontogenesis and tropopause undulation on the intensity, size, and structural changes of Hurricane Sandy (2012). J. Atmos. Sci., 74, 893-913, doi:10.1175/JAS-D-15-0362.1.

Sinclair, M. R., 2002: Extratropical transition of southwest Pacific tropical cyclones. Part I: Climatology and mean structure changes. Mon. Wea. Rev., 130, 590-609, doi:10.1175/ 1520-0493(2002)130<0590:ETOSPT > 2.0.CO;2.

Smith, M. B., R. D. Torn, K. L. Corbosiero, and P. Pegion, 2016: Ensemble variability in rainfall forecasts of Hurricane Irene (2011). 32nd Conf. on Hurricanes and Tropical Meteorology, San Juan, Puerto Rico, Amer. Meteor. Soc., 14B.2, https://ams.confex.com/ams/32Hurr/webprogram/ Paper293227.html.

Song, J., J. Han, and Y. Wang, 2011: Cyclone phase space characteristics of the extratropical transitioning tropical cyclones 
over the western North Pacific. Acta Meteor. Sin., 25, 78-90, doi:10.1007/s13351-011-0006-y.

-, R. Wu, W. Quan, and C. Yang, 2013: Impact of the subtropical high on the extratropical transition of tropical cyclones over the western North Pacific. Acta Meteor. Sin., 27, 476-485, doi:10.1007/s13351-013-0410-6.

Sun, Y., Z. Zhong, and Y. Wang, 2012: Kinetic energy budget of typhoon Yagi (2006) during its extratropical transition. $M e$ teor. Atmos. Phys., 118, 65-78, doi:10.1007/s00703-012-0200-1.

Thorncroft, C. D., and S. C. Jones, 2000: The extratropical transitions of Hurricanes Felix and Iris in 1995. Mon. Wea. Rev., 128, 947-971, doi:10.1175/1520-0493(2000)128<0947: TETOHF $>2.0 . \mathrm{CO} ; 2$.

Torn, R. D., J. S. Whitaker, P. Pegion, T. M. Hamill, and G. J. Hakim, 2015: Diagnosis of the source of GFS medium-range track errors in Hurricane Sandy (2012). Mon. Wea. Rev., 143, 132-152, doi:10.1175/MWR-D-14-00086.1.

Toth, Z., and E. Kalnay, 1997: Ensemble forecasting at NCEP and the breeding method. Mon. Wea. Rev., 125, 3297-3319, doi:10.1175/1520-0493(1997)125<3297:EFANAT >2.0.CO;2.

Uhlhorn, E. W., B. W. Klotz, T. Vukicevic, P. D. Reasor, and R. F. Rogers, 2014: Observed hurricane wind speed asymmetries and relationships to motion and environmental shear. Mon. Wea. Rev., 142, 1290-1311, doi:10.1175/MWR-D-13-00249.1.

Velden, C., and Coauthors, 2006: The Dvorak tropical cyclone intensity estimation technique: A satellite-based method that has endured for over 30 years. Bull. Amer. Meteor. Soc., 87, 1195-1210, doi:10.1175/BAMS-87-9-1195.

Vera, C., and Coauthors, 2006: Toward a unified view of the American monsoon systems. J. Climate, 19, 4977-5000, doi:10.1175/JCLI3896.1.

Veren, D., J. L. Evans, S. Jones, and F. Chiaromonte, 2009: Novel metrics for evaluation of ensemble forecasts of tropical cyclone structure. Mon. Wea. Rev., 137, 2830-2850, doi:10.1175/ 2009MWR2655.1.

Waliser, D. E., and Coauthors, 2012: The "Year" of Tropical Convection (May 2008-April 2010): Climate variability and weather highlights. Bull. Amer. Meteor. Soc., 93, 1189-1218, doi:10.1175/2011BAMS3095.1.

Walsh, K. J. E., and J. J. Katzfey, 2000: The impact of climate change on the poleward movement of tropical cyclone-like vortices in a regional climate model. J. Climate, 13, 1116-1132, doi:10.1175/1520-0442(2000)013<1116:TIOCCO > 2.0.CO;2.

Wang, Q., Q. Li, and G. Fu, 2012: Determining the extratropical transition onset and completion times of Typhoons Mindulle (2004) and Yagi (2006) using four methods. Wea. Forecasting, 27, 1394-1412, doi:10.1175/WAF-D-11-00148.1.

Weissmann, M., and Coauthors, 2011: The influence of assimilating dropsonde data on typhoon track and midlatitude forecasts. Mon. Wea. Rev., 139, 908-920, doi:10.1175/2010MWR3377.1. , R. H. Langland, C. Cardinali, P. M. Pauley, and S. Rahm, 2012: Influence of airborne Doppler wind lidar profiles near typhoon Sinlaku on ECMWF and NOGAPS forecasts. Quart. J. Roy. Meteor. Soc., 138, 118-130, doi:10.1002/qj.896.

Weng, Y., and F. Zhang, 2016: Advances in convection-permitting tropical cyclone analysis and prediction through EnKF assimilation of reconnaissance aircraft observations. J. Meteor. Soc. Japan, 94, 345-358, doi:10.2151/jmsj.2016-018.

Wolde, M., D. Marcotte, J. Jordan, J. Aitken, J. Abraham, and J. W. Strapp, 2001: The first Canadian experience with research flight operations in hurricane extratropical transition. Can. Aeronaut. Space J., 47, 179-189.

Wood, K. M., and E. A. Ritchie, 2012: The unusual behavior and precipitation pattern associated with Tropical Storm Ignacio (1997). Mon. Wea. Rev., 140, 3347-3360, doi:10.1175/ MWR-D-11-00284.1.

— impacts on the southwestern United States. Mon. Wea. Rev., 141, 4322-4336, doi:10.1175/MWR-D-13-00078.1.

— transition in the eastern North Pacific. J. Climate, 27, 59996015, doi:10.1175/JCLI-D-13-00645.1.

$\longrightarrow$, and $\longrightarrow$, 2014b: A 32-year reanalysis intercomparison of tropical cyclone structure in the eastern North Pacific and North Atlantic. 31st Conf. on Hurricanes and Tropical Meteorology, San Diego, CA, Amer. Meteor. Soc., 53, https:// ams.confex.com/ams/31Hurr/webprogram/Paper244195.html.

Wu, C. C., and Coauthors, 2005: Dropsonde Observations for Typhoon Surveillance near the Taiwan Region (DOTSTAR): An overview. Bull. Amer. Meteor. Soc., 86, 787-790, doi:10.1175/BAMS-86-6-787.

Yu, C.-K., and L.-W. Cheng, 2008: Radar observations of intense orographic precipitation associated with Typhoon Xangsane (2000). Mon. Wea. Rev., 136, 497-521, doi:10.1175/ 2007MWR2129.1.

— , and - 2013: Distribution and mechanisms of orographic precipitation associated with Typhoon Morakot (2009). J. Atmos. Sci., 70, 2894-2915, doi:10.1175/JAS-D-12-0340.1.

Zarzycki, C. M., D. R. Thatcher, and C. Jablonowski, 2017: Objective tropical cyclone extratropical transition detection in high-resolution reanalysis and climate model data. $J$. $A d v$. Model. Earth Syst., 9, 130-148, doi:10.1002/2016MS000775.

Zhang, F., and Y. Weng, 2015: Predicting hurricane intensity and associated hazards: A five-year real-time forecast experiment with assimilation of airborne Doppler radar observations. Bull. Amer. Meteor. Soc., 96, 25-32, doi:10.1175/ BAMS-D-13-00231.1.

Zhang, W., W. Perrie, and W. Li, 2006: Impacts of waves and sea spray on midlatitude storm structure and intensity. Mon. Wea. Rev., 134, 2418-2442, doi:10.1175/MWR3191.1.

Zhou, Y., and C. J. Matyas, 2017: Spatial characteristics of stormtotal rainfall swaths associated with tropical cyclones over the eastern United States. Int. J. Climatol., 37, 557-569, doi:10.1002/joc.5021. 